\title{
Sigma transformation and ALE formulation for three-dimensional free surface flows
}

\author{
Astrid Decoene, Jean-Frédéric Gerbeau ${ }^{\dagger}$ \\ ${ }^{*} M O X-$ Modelling and Scientific Computing \\ Dipartimento di Matematica "F. Brioschi", Politecnico di Milano \\ Via Bonardi 9, 20133 Milano, Italy \\ REO Project - INRIA Rocquencourt \\ BP 105, Le Chesnay 78153, France
}

\begin{abstract}
In this paper we establish the link between the sigma transformation approach and the ALE approach. For that purpose we introduce the ALE-Sigma (ALES) approach, which consists in an ALE interpretation of the sigma transformation. Taking advantage of this new approach, we propose a general ALES transformation, allowing for a great adaptability of the vertical discretization and therefore overcoming some drawbacks of the classical sigma transformation. Numerical results are presented, showing the advantages of this general coordinate system, as for example a better representation of horizontal stratifications.
\end{abstract}

Keywords: sigma transformation; ALE; free surface flows; horizontal pressure gradient error

\section{INTRODUCTION}

In this paper we focus on a particular way of treating the domain movement in three-dimensional free surface flows. Several techniques exist to deal with this critical feature. Among them, the Arbitrary Lagrangian Eulerian (ALE) approach - see for instance [13, 31] - has been adopted by many authors because of its great adaptability. It consists in moving the mesh at an arbitrary velocity which should be different from the fluid velocity, allowing for a continuous updating of the mesh. Another widely used technique, especially in the atmospheric and oceanographic communities, is referred to as the sigma transformation or topography-following coordinate system [26]. It consists in performing a transformation of the vertical coordinate - the $z$ coordinate - allowing to adopt a vertical discretization of the domain which at each time step follows the bathymetry and the free surface. Different systems have been developed on this approach, characterized by the choice of the discrete transformation function $[2,27,29,17]$.

To our knowledge, the link between both approaches is not clearly established in the literature. In the works describing the sigma transformation and its different variants, the ALE approach is generally not mentioned. In [32] an ALE formulation based on the classical variant of the sigma transformation for a shallow water flow problem is presented. It is claimed that

*astrid.decoene@mate.polimi.it

${ }^{\dagger}$ jean-frederic.gerbeau@inria.fr 
the conventional sigma models solve flow equations without accounting for the effect of moving grids in the vertical direction. In this paper we state the contrary, and one of our purposes is to show that the sigma transformation is equivalent to the ALE approach with a particular type of mappings. Therefore, we introduce the ALE-Sigma (ALES) approach, which consists in an ALE interpretation of the sigma transformation.

Our second purpose is to introduce a generalization of the classical sigma transformation taking advantage of its reinterpretation in the ALE frame. Although the sigma transformation technique presents many advantages - essentially the accurate assimilation of the bed and surface boundaries and the possibility of easily incorporating boundary layers - its classical variant, still widely used, presents important drawbacks. On one hand, it limits the possibilities of vertical discretization and therefore prevents from correctly adapting the mesh to the particular needs of the simulation. On the other hand, the classical sigma transformation can induce an important error in the computation of the horizontal pressure gradient in the presence of surface or bottom gradients. In particular, this error leads to an inaccurate representation of possible density-stratifications. We propose in this paper a general transformation in the ALES approach which allows for a great adaptability of the vertical discretization. The ALE-Sigma transformation allows to choose arbitrarily the layer distribution over the vertical of the domain, and in that way to overcome, when possible, the drawbacks of the classical sigma transformation, as we show through numerical tests at the end of this paper.

In the first section of this paper we present the sigma transformation approach, and we give a detailed description of the use and drawbacks of its most classical form. Then, we introduce the ALE-Sigma approach and we give a formulation of the three-dimensional free surface flow problem in this approach. A generalization of the sigma transformation in the ALES approach is given in the fourth section and some advantages of this generalization are illustrated numerically in the last section.

\section{THE SIGMA TRANSFORMATION APPROACH}

\subsection{Principle}

Let us first point out that this technique is applied to two- or three-dimensional domains in which the lateral boundaries are perfectly vertical - in the sequel we will consider three-dimensional domains. This configuration is widely used for ocean and coastal applications, but also in other shallow areas. Only the surface and bottom boundaries move, and they are described by two functions defined on the same two-dimensional domain. It is then possible to perform a variable change which transforms the vertical coordinate of each point in the real domain into a value contained in a fixed interval - for instance $[0,1]$ - depending on the time and on the position of this point in the two-dimensional domain. In fact, this comes to transform the irregular physical domain into a fixed regular reference configuration.

Before going further, let us describe more explicitly the considered configuration. Let $\omega$ be the invariant two-dimensional domain, whereas $\Omega_{t}$ denotes the three-dimensional domain at each time $t \in I$. The functions describing the free surface and the bottom of $\Omega_{t}$ are, respectively, $\eta(x, y, t)$ and $b(x, y, t)$, whilst $h(x, y, t)=\eta(x, y, t)-b(x, y, t)$ denotes the water depth. The current domain is then defined by

$$
\Omega_{t}=\{\boldsymbol{x}=(x, y, z) /(x, y) \in \omega \quad \text { and } \quad b(x, y) \leq z \leq \eta(x, y, t)\} .
$$


The sigma transformation approach consists in transforming the Cartesian coordinate system $(x, y, z, t)$ into a topography-following coordinate system $(\hat{x}, \hat{y}, \hat{z}, \hat{t})$, called $\sigma$-coordinate system and defined by

$$
\hat{t}=t, \quad \hat{x}=x, \quad \hat{y}=y \quad \text { and } \quad \hat{z}=\sigma(x, y, z, t),
$$

where $\sigma(x, y, \hat{z}, t)$ is an arbitrary continuous and monotonic $(\partial \sigma / \partial z \geq 0)$ function satisfying

$$
\left\{\begin{array}{l}
\sigma(x, y, \eta(x, y, t), t)=1 \\
\sigma(x, y, b(x, y, t), t)=0
\end{array} \quad \forall(x, y, t) \in \omega \times I .\right.
$$

The reference configuration is therefore:

$$
\hat{\Omega}=\{\hat{\boldsymbol{x}}=(\hat{x}, \hat{y}, \hat{z}) /(\hat{x}, \hat{y}) \in \omega \quad \text { and } \quad 0 \leq \hat{z} \leq 1\} .
$$

An example of such a transformation is shown in Figure 1.

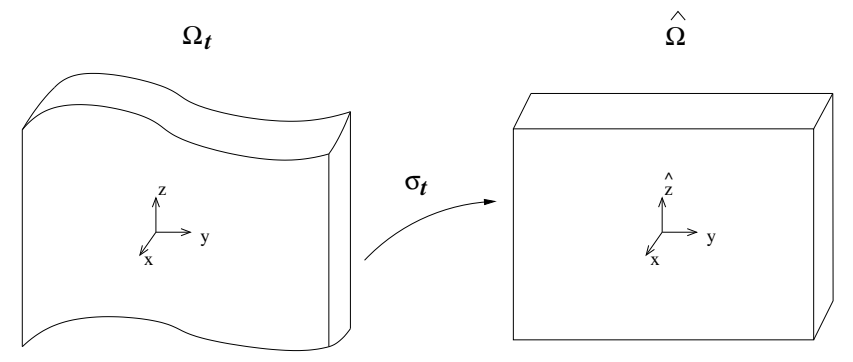

Figure 1: Example of a sigma transformation

Thus a particular transformation function $\sigma(x, y, \hat{z}, t)$ is chosen. Then, a triangulation of the invariant two-dimensional domain $\omega$ is defined and $\sigma(x, y, \hat{z}, t)$ is discretized. In some cases the transformation function is directly defined at the discrete level. The three-dimensional mesh $\hat{\mathcal{T}}_{h}$ is defined on the reference configuration $\hat{\Omega}$ by arbitrarily distributing the two-dimensional mesh as horizontal levels along the vertical. The corresponding mesh on the real domain is then defined at each time step through the discrete transformation function $\sigma_{h}(x, y, \hat{z}, t)$. Thus, the real mesh only moves in the vertical direction. For the sake of clarity, we indicate that, if $i$ denotes a particular node and $t^{n}$ denotes a particular time, then the coordinates of $i$ in the real domain at $t=t^{n}$ are

$$
x_{i}^{n}=\hat{x}_{i}, \quad y_{i}^{n}=\hat{y}_{i} \quad \text { and } \quad z_{i}^{n}=\sigma_{h}\left(\hat{x}_{i}, \hat{y}_{i}, \hat{z}_{i}, t^{n}\right),
$$

where $\left(\hat{x}_{i}, \hat{y}_{i}, \hat{z}_{i}\right)$ are the constant coordinates of $i$ in the reference configuration. The equations describing the flow are then completely written in the reference domain; more precisely all the partial derivatives are transformed into derivatives with respect to the $\sigma$-coordinates $(x, y, \hat{z})$.

The adoption of a $\sigma$-coordinate system allows an accurate and smooth definition of the bed and surface boundaries at each time. Indeed, the values of $\hat{z}$ at the bottom and the free surface are constant in time and space; that is, respectively, $\hat{z}=0$ and $\hat{z}=1$. This simplifies the kinematic boundary condition considerably both at the bottom and the free surface. In addition, one can easily incorporate bottom and surface boundary layers, and increase their resolution without having to increase the global number of layers. This is not possible in the commonly 
used $z$-coordinate system [3], in which the bottom profile is approximated by a staircase form. Such a step-discretization provokes a loss of accuracy in the assimilation of the bathymetry, as well as spurious errors. Many comparisons have been made between topography-following systems and the $z$-coordinate system (see for instance [10] and [25]), and these have shown that inferior numerical results are obtained with the second one, especially in the simulation of overflow processes and bottom layer dynamics.

For all these reasons, it has been gaining popularity and is used for a very large range of ocean and coastal applications - see for instance [2, 27, 29] -, and has more recently also been applied to basin-scale problems [12, 17, 32, 34] and even sea-ice models [14]. The simplest and most commonly used $\sigma$-coordinate system is the one introduced by Phillips in [26], to which we will refer to as the classical sigma transform.

\subsection{The classical sigma transformation and its drawbacks}

The classical $\sigma$-coordinate system [26] consists in adopting the following $\sigma$ function:

$$
\sigma(x, y, z, t)=\frac{z-b(x, y, t)}{h(x, y, t)} .
$$

In this configuration the z-coordinates of the mesh nodes in the real domain are incremented of $b_{h}(x, y, t)$ and multiplied by the coefficient $h_{h}(x, y, t)$ relatively to their value in the reference domain. Moreover the relative width of each layer - i.e. the width divided by the local water depth - is equal to the corresponding width in the reference configuration, and thus it is constant over the time and over the whole two-dimensional domain $\omega$. Figure 2 shows an example of such a mapping.

$\hat{\Omega}$

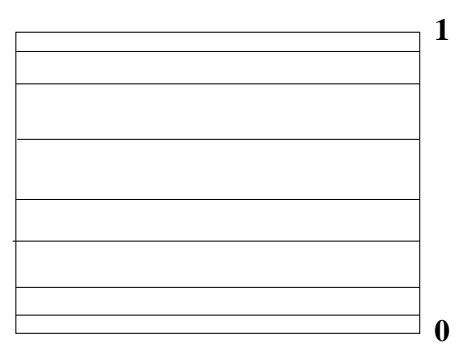

1

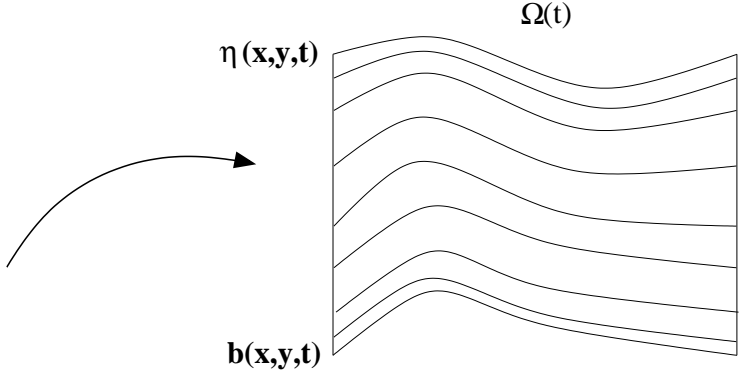

Figure 2: Example of a classical sigma-transformation

The advantage of this coordinate system is its simplicity: not only the implementation of the transformation is easy, but the formulas for the variable change between the real and the reference framework are relatively simple. Indeed the determinant of the transformation is equal to the water depth function

$$
\hat{J}_{t}=\frac{\partial z}{\partial \hat{z}}=h(x, y, t) \text {. }
$$

However, the classical sigma-transformation also presents major drawbacks, due to the restriction it operates on the vertical discretization. Indeed, it implies that all the levels necessarily follow the bottom and free surface functions, and that the relative width of each layer - i.e. the width divided by the local water depth - is constant in time and over the whole two-dimensional 
domain. As a consequence, it cannot maintain equally high resolution in particular zones - for instance near the surface and the bottom - independently of the local water depth. Now many applications need a high resolution of the surface and bottom layers everywhere, for instance to represent accurately a surface mixing process arising from wind, thermal forcing and turbulence. More generally, with a constant number of horizontal levels, deeper zones of the domain can be under-discretized and shallower zones over-discretized, and the latter may cause severe CFL restrictions.

In addition, the classical sigma-transformation prevents from fixing the mesh in a particular zone of the domain which shall be solved more accurately. Now the movement of the mesh can generate an amplification of the numerical diffusion, leading to a relevant perturbation of the solution. This feature is illustrated through a simple numerical test case in the last section of this paper.

Finally, the classical sigma-transformation can induce the so-called sigma-coordinate pressure gradient error $[9,15]$, which has evoked considerable concern in the meteorological and oceanographic communities $[1,5,11,23,30,33]$. It has been recognized that, in the presence of bottom or free surface gradients, the topography-following shape of the mesh can yield significant errors in the computation of the horizontal pressure gradient (HPG) in the momentum equation. Indeed, owing to the sigma-transformation, the grid elements become non-orthogonal and may even deteriorate quite strongly in shallow areas - for instance near tidal flats. Now this can lead to errors in the computation of the horizontal gradient of a $z$-dependent function, if this function is not approximated accurately enough. Especially, the HPG error can be relevant in density-stratified flows. Let us next describe more precisely the root and effects of this sigma-coordinate pressure gradient error.

\subsection{The sigma-coordinate pressure gradient error}

This problem has essentially been analysed in the framework of hydrostatic flows, where the pressure gradient error concerns the buoyancy term - that is the term involving the spatial density variations -, because it involves the horizontal gradient of a $z$-dependent function. More particularly, the HPG error arises from the barotropic part of the buoyancy term, which writes:

$$
g \nabla_{2}\left(\int_{z}^{\eta} \frac{\Delta \rho}{\rho_{0}} d \delta\right)
$$

where $\Delta \rho$ is the fluctuation of the fluid density around an average value $\rho_{0}$ and $\nabla_{2}$ denotes the horizontal gradient. This term accounts for the accelerations arising from the slight density variations in the fluid.

The main difficulty in computing this term is attributed to the deformation of the grid cells in the presence of bottom or surface gradients. Such a situation is especially dangerous for the finite difference models because of the truncation errors inherent to the method. Besides, most authors have analysed this problem for the case of a finite difference discretization - see for instance [23, 30,33]. They point out that the truncation errors in the computation of the horizontal gradient are increased by the transformation of the horizontal gradient in the sigma-coordinate system. In the worst case, the deterioration of the grid cells can render the numerical scheme "hydrostatically inconsistent". This means that the difference between the discrete and the analytical version of the HPG force does not decrease when the resolution is increased, and that occurs when the so-called hydrostatic consistency condition (see [15] and [11]) is violated. Typically this is the case when, in presence of very steep bottom or free surface 
gradients, one or more of the nodes at the bottom of a real mesh element are situated above some nodes at its top, as illustrated by Figure 3 for prismatic elements. Such a situation can
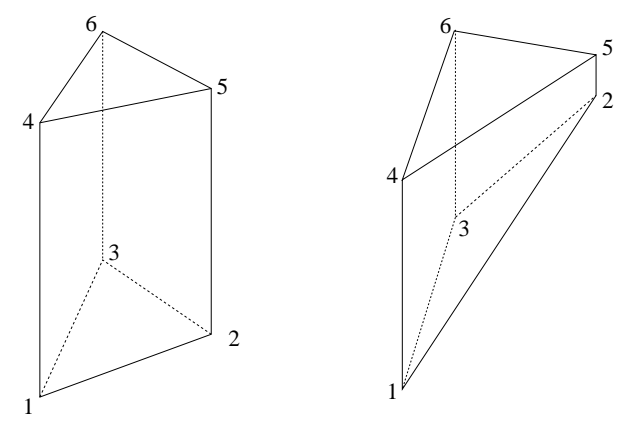

Figure 3: Example of hydrostatically consistent (left) and inconsistent (right) elements.

sometimes be avoided already during mesh generation by finer horizontal discretization or lower vertical discretization, especially when it is only caused by the bottom gradient. Unfortunately, as pointed out by Mellor et al. in [23], the hydrostatic consistency condition is quite difficult to fulfil in oceanic applications and especially for estuaries.

In the case of a finite element method, the errors are of smaller magnitude because the pressure gradient can be computed to a higher order. However, the HPG error exists and can be relevant, even in geometries where the hydrostatic consistency condition is fulfilled. It arises essentially from the interpolation error on the relative density field, which can be amplified when computing its gradient. Especially, it becomes difficult to catch a density-stratification in deformed elements when the interpolation functions are not of high order.

An error in the computation of the horizontal pressure gradient can be easily detected in test cases where the density field varies only in the vertical direction - due for instance to a horizontal stratification of the temperature or the water salinity. In this cases, the barotropic buoyancy source term (8) is zero :

$$
g \nabla_{2}\left(\int_{z}^{\eta} \frac{\Delta \rho(\delta)}{\rho_{0}} d \delta\right)=0
$$

In absence of exterior source terms, the configuration should theoretically produce zero velocities: this makes spurious velocities easy to detect. Moreover, the density field should remain unchanged, a perturbation of the stratification can therefore illustrate the error. Many models using the classical sigma-transformation have been tested on such cases and they produce artificial flows, sometimes even degrading the solution beyond an acceptable limit (see in particular the results obtained on the idealized sea-mount test case $[1,5,24])$. It has been shown that the pressure gradient error depends on the coordinate slope, the density gradient and the polynomial interpolation degree of the vertical density distribution. Larger errors are found in the areas where the pycnoclines intersect the bottom slope, and especially in shallower zones.

Simulations performed with the finite-element-based, three-dimensional free surface flow solver Telemac-3d [12], in regions with non-horizontal bottom have also shown important errors in cases where strong stratifications are present [20]. Moreover, they appear even when the hydrostatic consistency condition is fulfilled. Simple test cases have shown that these errors 
arise from the interpolation errors on the buoyancy term [20,21] inherent to the finite element method. In order to clarify this matter, let us develop the case where the density field varies quadratically in the vertical direction only, for instance:

$$
\Delta \rho(x, y, z, t)=a z^{2}+b \text { in } \Omega_{t} \times I,
$$

where $a$ and $b$ are two constants. At the continuous level we have that

$$
\nabla_{2}\left(\int_{z}^{\eta} \frac{\Delta \rho}{\rho_{0}} d \delta\right)=0
$$

Assume now that the density variations are interpolated by finite element functions which are piecewise linear in the vertical direction. At the discrete level, using the trapezium rule for the vertical integration - which is exact for linear functions-, and denoting by $N_{l}$ the number of levels in the mesh, we then have that:

$$
\begin{aligned}
\nabla_{2}\left(\int_{z}^{\eta_{h}} \frac{\Delta \rho_{h}}{\rho_{0}} d z\right) & =\frac{1}{\rho_{0}} \nabla_{2}\left(\sum_{k=1}^{N_{l}-1} \Delta z_{k+1 / 2} \frac{\Delta \rho\left(z_{k}\right)+\Delta \rho\left(z_{k+1}\right)}{2}\right) \\
& =\frac{1}{\rho_{0}} \nabla_{2}\left(\sum_{k=1}^{N_{l}-1} \Delta z_{k+1 / 2} \frac{a\left(z_{k}^{2}+z_{k+1}^{2}\right)+2 b}{2}\right) \\
& =\frac{1}{\rho_{0}} \sum_{k=1}^{N_{l}-1}\left(\frac{a\left(z_{k}^{2}+z_{k+1}^{2}\right)+2 b}{2} \nabla_{2} \Delta z_{k+1 / 2}\right) .
\end{aligned}
$$

where $z_{k}$ is the height of the $k$-th level and $\Delta z_{k+1 / 2}=z_{k+1}-z_{k}$, for $k=1, \ldots, N_{l}$. Now in zones where the grid elements are non-orthogonal, the horizontal gradient of the depth $\Delta z_{k+1 / 2}$ between the layers is not zero. In addition, because of the interpolation error on $\Delta \rho$, the terms do not compensate. Therefore, the value of the buoyancy term is non-zero, which causes spurious numerical density currents. This acts like an artificial source term, creating spurious velocities, and if the error is important, like in shallow areas with steep bottom slopes, it can disturb the stratifications and even the free surface.

Let us now illustrate this numerical phenomenon through some results obtained on the simple test case of a standing basin using the Telemac-3d system. We consider a closed rectangular basin with a length of $500 \mathrm{~m}$ and a width of $100 \mathrm{~m}$, and with a non-horizontal bottom varying quadratically between $-25 \mathrm{~m}$ and $-50 \mathrm{~m}$. The three-dimensional mesh is represented in Figure 4. It has been obtained using the classical sigma transformation and piling up the two-dimensional grid at 11 homogeneously distributed levels on the vertical. Its vertical crosssection at $y=50 \mathrm{~m}$ is shown in Figure 5. Note that this mesh fulfills the hydrostatic consistency condition.

The basin is filled with a fluid containing an active tracer $T$, acting on the density $\rho$ following the linear state equation :

$$
\rho=\rho_{0}+0.749979 c,
$$

where $c$ is the concentration of the tracer $T$. The initial density field can therefore be imposed by setting a particular initial distribution of the tracer. We make the relative density $\Delta \rho / \rho_{0}$ vary in the vertical direction only. We indicate that the density variation is interpolated linearly 


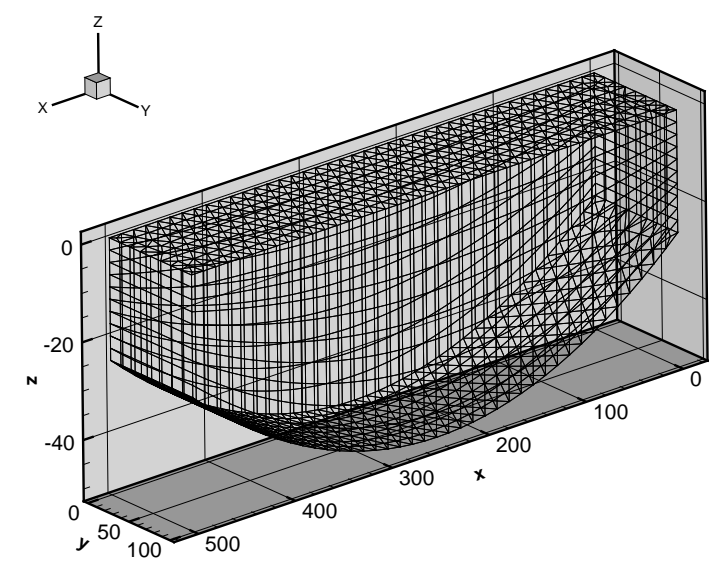

Figure 4: The initial 3D mesh.

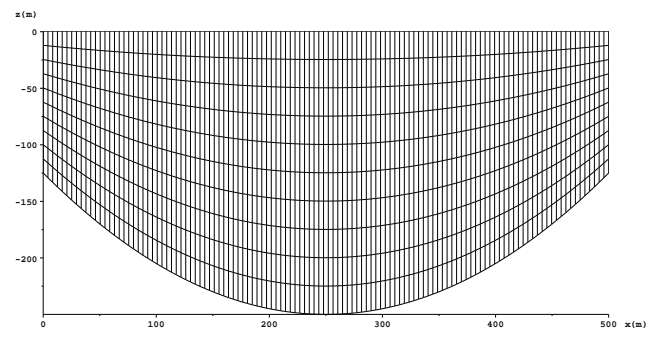

Figure 5: Vertical cross-section of the initial 3D mesh at $y=50 \mathrm{~m}$.

in the Telemac-3d system. At the initial time, the free surface is constant and the velocity of the fluid is zero. In addition, the diffusivity of the tracer is neglected and no exterior source term is applied. The analytical solution of this test is thus clear and simple: the free surface and the density field remain undisturbed, and the fluid velocity is zero.

We emphasize on the fact that this test is relevant for applications in hydraulics. In fact, it can be seen as a simplified configuration of a lake or an ocean at equilibrium. And it is essential that the numerical scheme preserves this equilibrium, in order to be able to simulate more complex and realistic cases. This test case is similar to the well-known idealized sea-mount test case $[1,5,24]$ used by many authors when dealing with the horizontal pressure gradient error. However, the sea-mount test involves a very steep topography. We show with our test that important errors can also appear in the presence of milder bottom gradients.

First, we set a density field varying linearly in the vertical direction only. The initial density distribution, as well as the distribution after 100 seconds are presented in Figure 6. Since the density is interpolated linearly, the horizontal pressure gradient is computed exactly. Indeed, as expected, the system remains unchanged and no spurious velocities are detected.

The initial tracer distribution is then modified so that the density field varies quadratically in the vertical direction. The degree of the density variation is now higher than the degree of the 

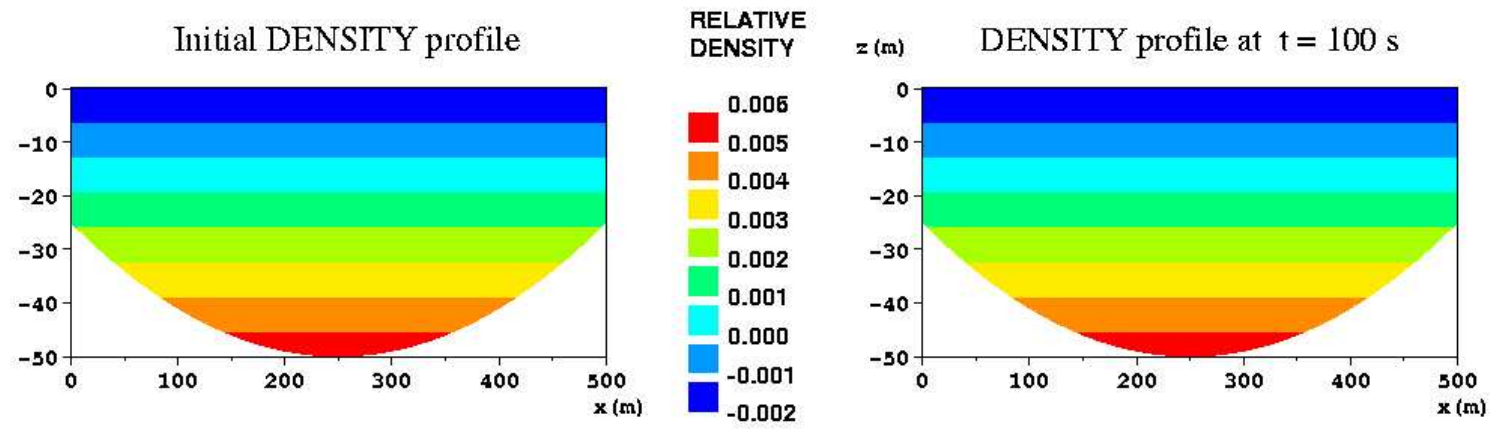

Figure 6: Initial linear density distribution and distribution obtained at $t=100$ s. Vertical cross-section at $y=50 \mathrm{~m}$.

interpolation, and this leads to an error in the computation of the horizontal pressure gradient. The initial density distribution, as well as the distribution after 100 seconds are presented in Figure 7. The HPG error has no impact on the density distribution, but it provokes the creation
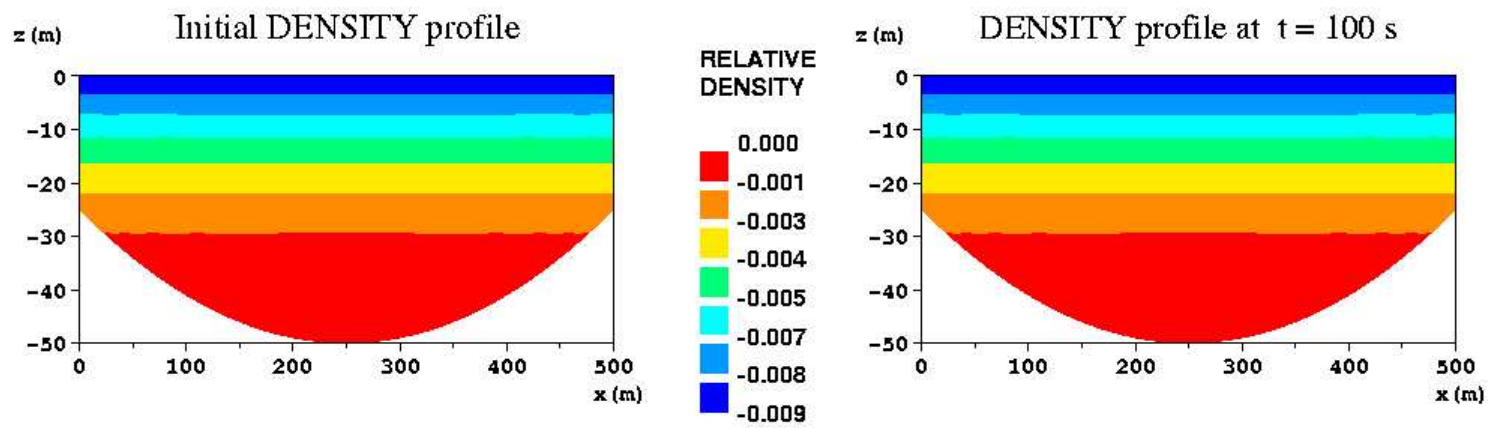

Figure 7: Initial quadratic density distribution and distribution obtained at $t=100$ s. Vertical cross-section at $y=50 \mathrm{~m}$.

of spurious velocities. We can evaluate the error $e_{h}$ of the simulation as the Euclidian norm of the spurious velocity:

$$
e_{h}=\left\|\boldsymbol{U}_{h}\right\|
$$

Figure 8 shows the profile of this error $e_{h}$ as a function of the basin depth at $x=100 \mathrm{~m}$, where the error is the most significant. The maximal values of the error are obtained at the bottom and at the free surface of the basin, where it reaches $2.0410^{-3} \mathrm{~m} / \mathrm{s}$.

The spurious velocities increase significantly when a stratified density field is set. In the next test, $\rho$ is piecewise constant and discontinuous at a horizontal interface - as it happens for instance in presence of a horizontal temperature or salinity stratification. The initial density stratification is presented left in Figure 9 and the density distribution obtained at $t=100 \mathrm{~s}$ right on the same figure: the density stratification has been significantly perturbed. In this case, the HPG error creates larger spurious velocities which perturb the density stratification. Figure 10 presents the profile of the error $e_{h}$ at $x=100 \mathrm{~m}$ : at the surface of the basin it reaches $0,12 \mathrm{~m} / \mathrm{s}$. 


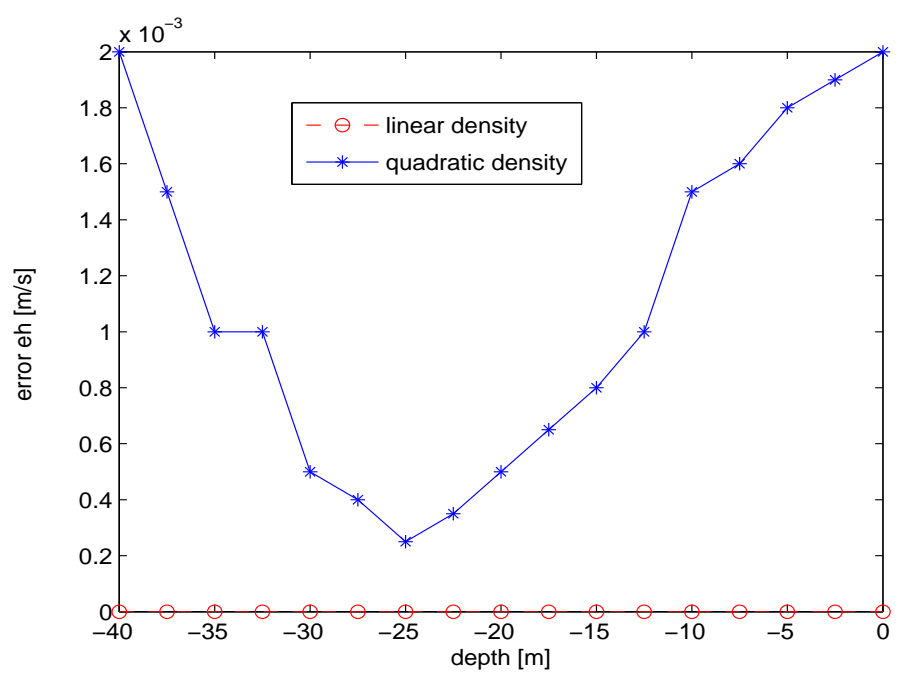

Figure 8: Profile of the error $e_{h}$ as a function of the basin depth at $x=100 \mathrm{~m}$ and $t=100 \mathrm{~s}$.
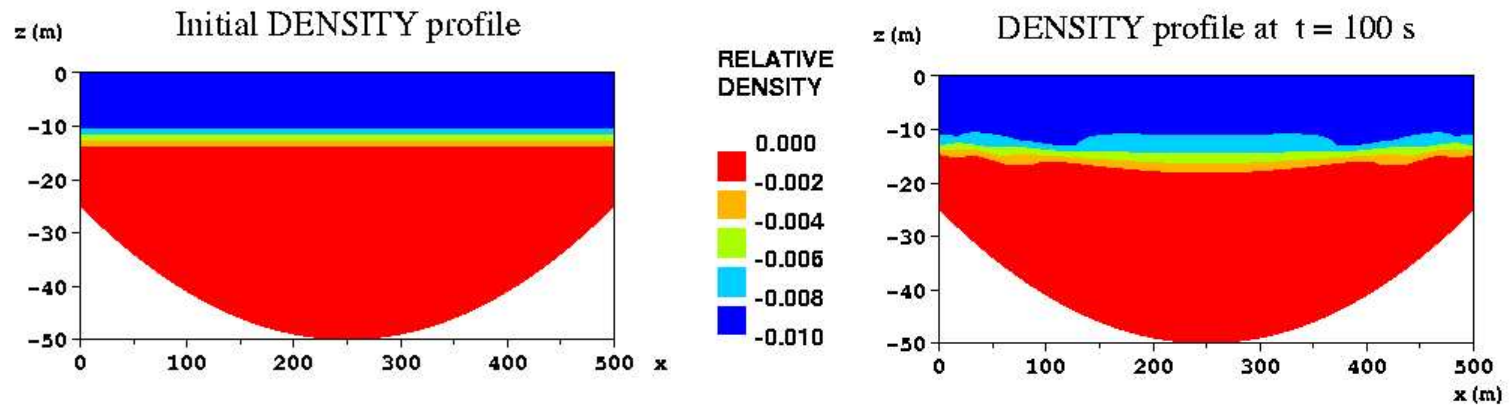

Figure 9: Initial density stratification and density distribution at $\mathrm{t}=100 \mathrm{~s}$. Vertical cross-section at $y=50 \mathrm{~m}$.

If we go further with the simulation, we notice that the system tends to a new steady state, in which the tracer distribution becomes smooth, so that the HPG error decreases. Figure 11 shows the time evolution of the error $E_{h}(t)$, defined as the $L^{2}$ norm of the spurious velocity:

$$
E_{h}(t)=\left(\int_{\Omega(t)}\|\boldsymbol{U}\|^{2}\right)^{1 / 2} .
$$

The error oscillates, as a consequence of the evolution of the density stratification, and seems to converge to a non zero value. Figure 12 shows the density distribution obtained after 1000 seconds. As we can see, the horizontal stratification is now almost smooth. The gradient of the density variation has therefore decreased, and so has also the error in the computation of the horizontal pressure gradient. As a consequence, the spurious velocities are smaller, as shown in figure 13. The maximal value of the local error $e_{h}$ is now $6.10^{-2} \mathrm{~m} / \mathrm{s}$. However, since the density distribution does not become linear, the error does not cancel.

This test cases clearly reveal the appearance of an HPG error when the density field is not interpolated exactly in the finite element method. In addition, they show that this error 


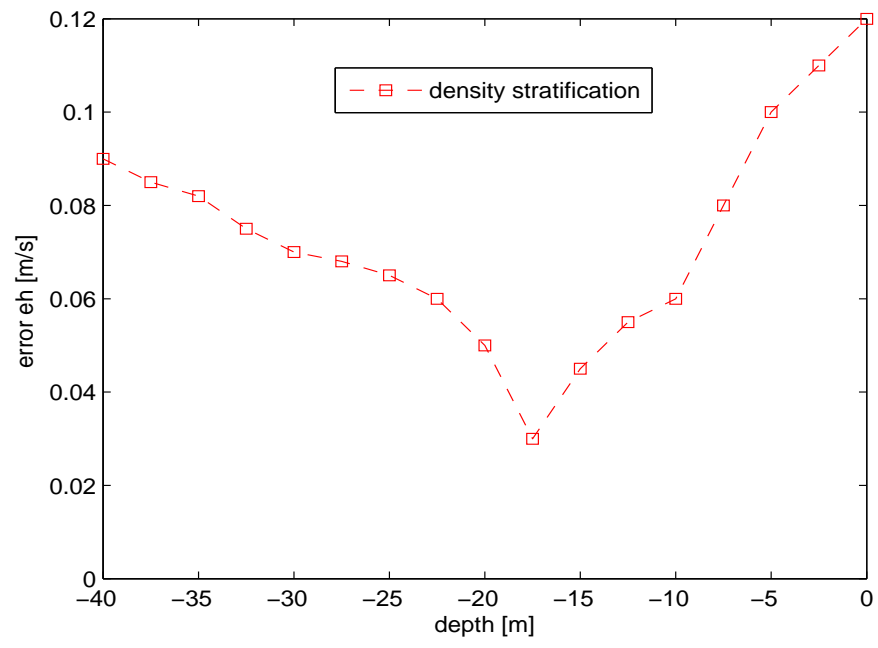

Figure 10: Profile of the error $e_{h}$ as a function of the basin depth at $x=100 \mathrm{~m}$ and $t=100 \mathrm{~s}$ with an initial density stratification.

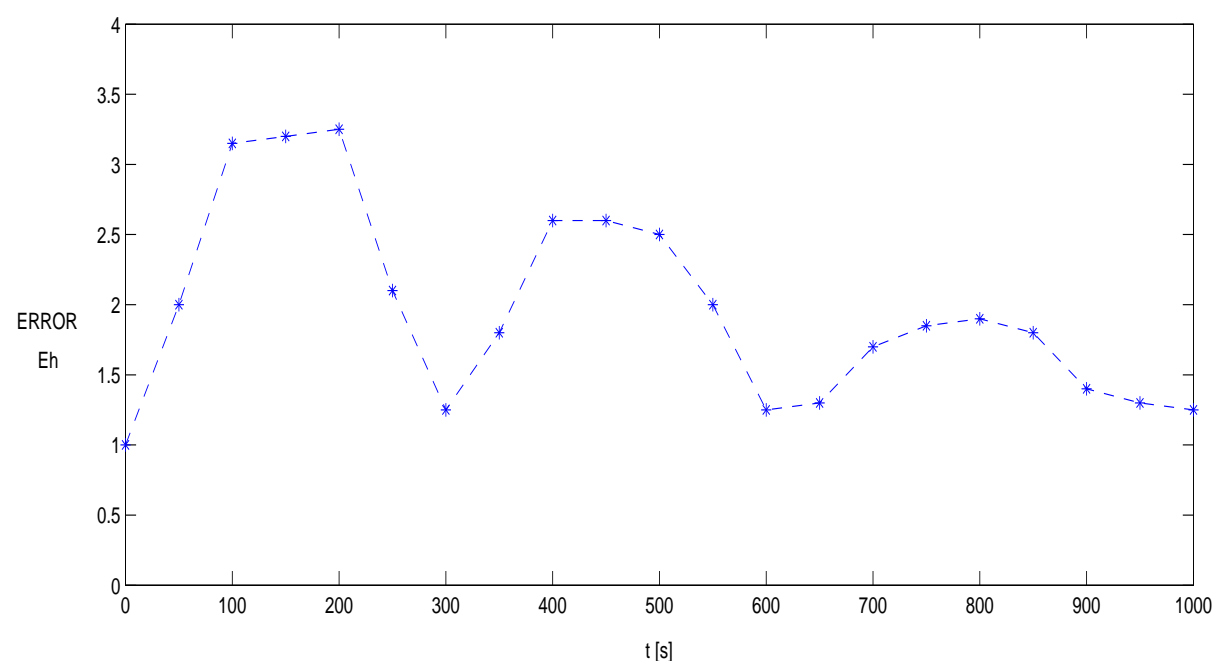

Figure 11: Time evolution of the error $E_{h}$ in the case of an initial horizontal density stratification with the classical sigma mesh.

increases with the value of the initial vertical density gradient. Indeed, the maximal spurious velocities are obtained when the density field is discontinuous, thus when its vertical gradient is infinite. On the other hand, they appear even when the hydrostatic consistency condition is fulfilled, and they can be significant over bottoms with mild gradients.

Since the stratifications are nearly horizontal in most situations, a $z$-coordinate system might be preferred, at least locally. But for applications where both the topography and the density are essential, this wouldn't be a valid option. Another system avoiding the pressure gradient problem is the so-called isopycnic coordinate system, which uses the density as the 


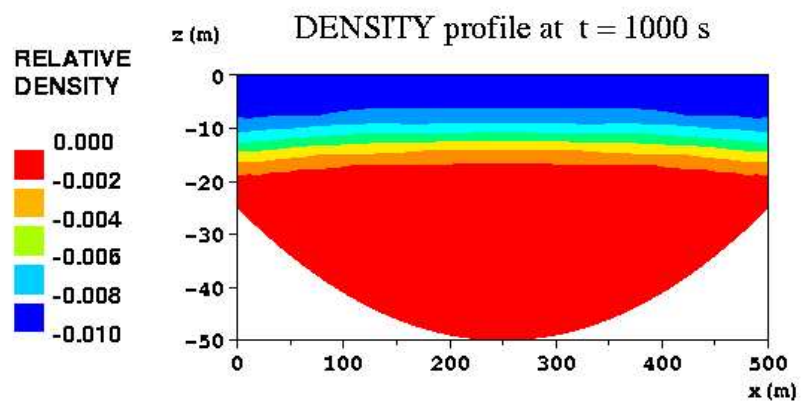

Figure 12: Density distribution at $\mathrm{t}=1000 \mathrm{~s}$. Vertical cross-section at $y=50 \mathrm{~m}$.

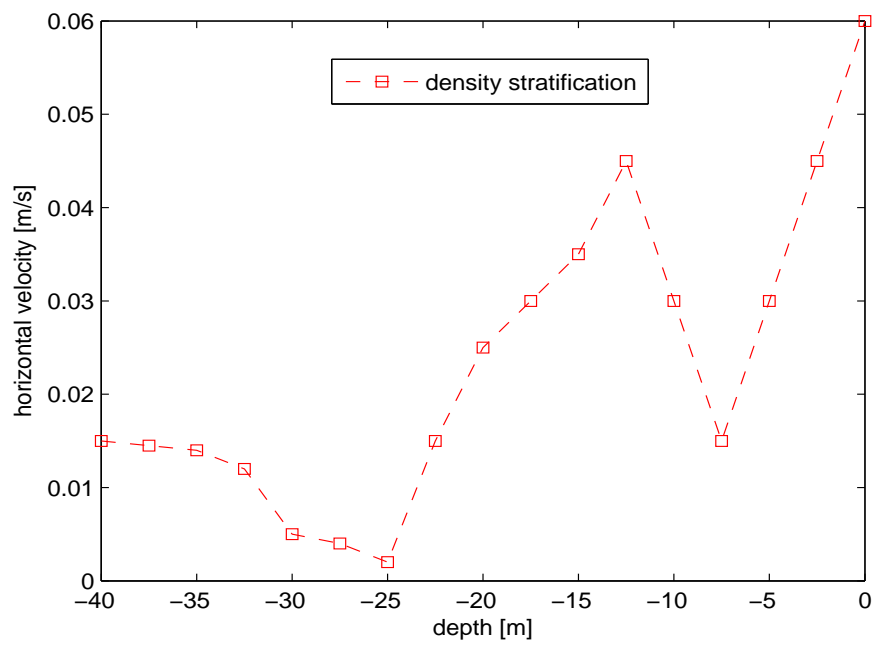

Figure 13: Profile of the error $e_{h}$ as a function of the basin depth at $x=100 \mathrm{~m}$ and $t=1000 \mathrm{~s}$ with an initial density stratification.

vertical coordinate. But this system is not adequate to study barotropic flows or whenever topography plays a major role.

Therefore, important efforts have been invested in the development of numerical methods improving the approximation of horizontal gradients. A simple and significant improvement is obtained by subtracting the horizontal mean value from the actual density field before computing the HPG force [23]. A further improvement results from the use of the so-called densityJacobian formulation of the pressure gradient (see [2] and [30]). It is obtained by applying the Leibniz rule to the baroclinic part of the buoyancy term (8):

$$
g \nabla_{2}\left(\int_{z}^{\eta} \frac{\Delta \rho}{\rho_{0}} d \delta\right)=g \int_{z}^{\eta} \nabla_{2}\left(\frac{\Delta \rho}{\rho_{0}}\right) d \delta+g \frac{\Delta \rho}{\rho_{0}} \nabla_{2} \eta .
$$

The density fluctuation term is differentiated first and integrated after, what allows a more accurate approximation. For instance, if the density field varies linearly, its vertical integration results in a second-order term. Thus, a second-order accurate differencing method has to be used to achieve an exact computation of the gradient using the expression left in (12). On the contrary, if the expression right in (12) is used, basic first-order derivation and second-order 
integration methods can be used. This technique has been convincing for the finite element system Telemac-3D - see [16] - in the case of a linear density fluctuation $\Delta \rho$. However, for non-linear or discontinuous density fields, errors still appear. Other techniques consist in implementing higher-order methods to estimate the pressure gradient term (e.g. [1] and [22]), but they fail in some cases such as in presence of strongly-stratified flows. Stelling and Van Kester [33] suggested an alternative method, which basically transforms the sigma-grid back to a Cartesian system before computing the HPG force, resulting in substantial reduction of the errors. This method, slightly improved in [28], is efficient but very costly. Moreover, substantial errors remain when there are strong vertical gradients in the density field.

An intensive research is still in course for developing new and more accurate methods for approximating horizontal gradients (see e.g. [5, 27]). But another way to overcome the problem is to design more general coordinate systems, combining the advantages of both the topographyfollowing and the $z$-coordinate systems. Such a system should allow a transition from sigma- to $z$-coordinate, such that the upper and lower layers follow, respectively, the free surface and the bottom topography, and layers in between are arbitrary. This would allow for more adaptability of the vertical discretization. For instance, in the case of the standing basin just presented, we can think of imposing a fixed horizontal plane at the level of the density stratification. Indeed, in this way, the density gradient would be computed on orthogonal grid elements, and the HPG error would cancel. However, this is not possible with the classical sigma transformation. A more general transformation is needed.

Let us now introduce the ALES approach, which is a reinterpretation of the sigma transformation in the ALE approach. This approach will then allow to define a very general sigmacoordinate system.

\section{THE ALE-SIGMA APPROACH}

\subsection{ALE interpretation of the sigma transformation}

Our purpose here is to show that the sigma transformation can be interpreted as the adoption of a particular ALE mapping and an unusual ALE formulation.

On one hand, the vertical-coordinate transformation (2)-(3) can be performed using the following ALE mapping between the reference configuration $\hat{\Omega}$ and the real domain $\Omega_{t}$ :

$$
\begin{aligned}
& \hat{\mathcal{A}}_{t}: \hat{\Omega} \longrightarrow \Omega_{t}, \quad \boldsymbol{x}(\hat{\boldsymbol{x}}, t)=\hat{\mathcal{A}}_{t}(\hat{\boldsymbol{x}}) \\
& \text { with } \quad x=\hat{x}, \quad y=\hat{y} \quad \text { and } \quad z=Z(x, y, \hat{z}, t),
\end{aligned}
$$

where $Z(x, y, \hat{z}, t)$ is an arbitrary continuous and monotonic $(\partial Z / \partial \hat{z} \geq 0)$ function satisfying

$$
\left\{\begin{array}{l}
Z(x, y, 0, t)=\eta(x, y, t), \quad \forall(x, y, t) \in \omega \times I, \\
Z(x, y, 1, t)=b(x, y, t) .
\end{array}\right.
$$

Actually, this definition includes the ALE mappings for domains moving only in the vertical direction. Note that consequently the domain velocity has only a vertical non zero component, that is $\boldsymbol{c}=(0,0, c)^{T}$.

On the other hand the sigma transformation approach states that the equations be completely transformed into the $\sigma$-coordinate system. That means that all partial derivatives are 
reformulated with respect to the fixed domain. Interpreting the sigma transformation as an ALE mapping makes clear that this consists in reformulating the derivatives into the ALE frame. Especially, the time derivatives are reformulated into ALE time derivatives: the effects of the moving domain are thus effectively taken into account.

Throughout, we will refer to the ALE interpretation of the sigma transformation as the ALE-Sigma (ALES) approach. We want to emphasize on the fact that the equations can be written both in the ALE frame or in the real domain, but it is essential that the time derivative be written in the ALE frame, so that the domain movement is taken into account. We introduce next an ALES formulation of the three-dimensional free surface flow problem, which is a combination of the usual ALE formulation and the one generally used in the sigma transformation approach.

\subsection{ALES formulation of the three-dimensional free surface flow problem}

Let us first introduce some relations involving the Jacobian determinant $\hat{J}_{t}$ of the mapping, as well as several derivation formulas that will be needed to write the equations in the reference configuration $\hat{\Omega}$. Since the mapping only transforms the vertical coordinates of the system, the continuous expression of the Jacobian determinant is

$$
\hat{J}_{t}(x, y, \hat{z}, t)=\frac{\partial Z}{\partial \hat{z}}(x, y, \hat{z}, t) .
$$

By a classical abuse of notation, we denote the time derivative on the ALE frame, written in the spatial coordinate, by $\left.\frac{\partial \psi}{\partial t}\right|_{\hat{\boldsymbol{x}}}$. It is defined as follows:

$$
\left.\frac{\partial \psi}{\partial t}\right|_{\hat{\boldsymbol{x}}}: \Omega_{t} \times I \longrightarrow \mathbb{R},\left.\quad \frac{\partial \psi}{\partial t}\right|_{\hat{\boldsymbol{x}}}(\boldsymbol{x}, t)=\frac{\partial \hat{\psi}}{\partial t}(\hat{\boldsymbol{x}}, t) \quad \text { with } \hat{\boldsymbol{x}}=\mathcal{A}_{t}^{-1}(\boldsymbol{x}) .
$$

The relation between the ALE time derivative of a function $\psi: \Omega_{t} \longrightarrow \mathbb{R}$ and its Eulerian time derivative is

$$
\frac{\partial \psi}{\partial t}=\left.\frac{\partial \psi}{\partial t}\right|_{\hat{\boldsymbol{x}}}+\frac{\partial \hat{z}}{\partial t} \frac{\partial \psi}{\partial \hat{z}}
$$

The following formulas relate the different space partial derivatives of $\psi$ :

$$
\begin{aligned}
\frac{\partial \psi}{\partial x} & =\left.\frac{\partial \psi}{\partial x}\right|_{\hat{\boldsymbol{x}}}+\frac{\partial \hat{z}}{\partial x} \frac{\partial \psi}{\partial \hat{z}}, \\
\frac{\partial \psi}{\partial y} & =\left.\frac{\partial \psi}{\partial y}\right|_{\hat{\boldsymbol{x}}}+\frac{\partial \hat{z}}{\partial y} \frac{\partial \psi}{\partial \hat{z}}, \\
\frac{\partial \psi}{\partial z} & =\frac{\partial \hat{z}}{\partial z} \frac{\partial \psi}{\partial \hat{z}} .
\end{aligned}
$$

Taking $\psi(x, y, z, t)=z$, essential relations can be derived:

$$
\begin{aligned}
\frac{\partial \hat{z}}{\partial t}=-\left.\hat{J}_{t}^{-1} \frac{\partial z}{\partial t}\right|_{\hat{\boldsymbol{x}}}, & \frac{\partial \hat{z}}{\partial x}=-\left.\hat{J}_{t}^{-1} \frac{\partial z}{\partial x}\right|_{\hat{\boldsymbol{x}}} \\
\frac{\partial \hat{z}}{\partial y}=-\left.\hat{J}_{t}^{-1} \frac{\partial z}{\partial y}\right|_{\hat{\boldsymbol{x}}}, & \frac{\partial \hat{z}}{\partial z}=\hat{J}_{t}^{-1} .
\end{aligned}
$$


We consider the Navier-Stokes equations describing the mean motion of an incompressible Newtonian fluid in a three-dimensional moving domain $\Omega_{t}$ of the type defined by (1). In the Cartesian frame, these equations are the following, valid for all $t \in I$ and for all $\boldsymbol{x} \in \Omega_{t}$ :

$$
\begin{aligned}
\frac{d \boldsymbol{u}}{d t}-\operatorname{div}(\nu \nabla \boldsymbol{u})+\frac{1}{\rho} \nabla_{2} p & =\boldsymbol{f}, \\
\frac{d w}{d t}-\operatorname{div}(\nu \nabla w)+\frac{1}{\rho} \frac{\partial p}{\partial z} & =-g, \\
\operatorname{div} \boldsymbol{U} & =0,
\end{aligned}
$$

where $\boldsymbol{u}$ and $w$ are respectively the horizontal and vertical velocities, $\boldsymbol{U}=(\boldsymbol{u}, w)^{T}$ is the total velocity, and $\nu$ is the dynamic viscosity coefficient of the fluid. The horizontal source term is denoted by $f$ and the gravity acceleration by $g$. This system is closed by convenient boundary conditions. In particular, the kinematic condition on the bottom of the domain $\Gamma_{b}$ expressing imperviousness is

$$
\boldsymbol{U} \cdot \boldsymbol{n}=0 \quad \text { on } \quad \Gamma_{b} \cup \Gamma_{b},
$$

where $\boldsymbol{n}$ is the outward normal to the current domain $\Omega_{t}$. At the free surface $\Gamma_{s, t}$, the velocity of the fluid is considered equal to the velocity of the free surface itself, which is expressed by the following kinematic condition:

$$
\frac{\partial \eta}{\partial t}-\boldsymbol{U} \cdot \boldsymbol{n}=0 \quad \text { on } \quad \Gamma_{s, t}
$$

The usual ALE formulation consists in transforming the eulerian time derivative in an ALE time derivative:

$$
\begin{aligned}
\left.\frac{\partial \boldsymbol{u}}{\partial t}\right|_{\hat{\boldsymbol{x}}}+\left(\boldsymbol{U}-\frac{\partial \hat{\boldsymbol{x}}}{\partial t}\right) \cdot \boldsymbol{\nabla} \boldsymbol{u}-\operatorname{div}(\nu \boldsymbol{\nabla} \boldsymbol{u})+\frac{1}{\rho} \nabla_{2} p & =\boldsymbol{f}, \\
\frac{d w}{d t}-\operatorname{div}(\nu \boldsymbol{\nabla} w)+\frac{1}{\rho} \frac{\partial p}{\partial z} & =-g, \\
\operatorname{div} \boldsymbol{U} & =0,
\end{aligned}
$$

The usual sigma transformation formulation consists in reformulating all the time and spatial derivatives into ALE frame. Here we will not transformation the second order derivatives in the diffusion terms, because this would lead to a very long and complex expression. In addition, it has been established (see e.g. [23, 30]) that a transformation of the horizontal pressure gradient into a topography-following coordinate system can increase the computational error of this term. We therefore also keep the partial derivatives in this terms in the Cartesian frame. The definition of a new "pseudo vertical velocity" simplifies the transformation of the equations:

$$
w^{*}=\frac{d \hat{z}}{d t}=\frac{\partial \hat{z}}{\partial t}+u \frac{\partial \hat{z}}{\partial x}+v \frac{\partial \hat{z}}{\partial y}+w \frac{\partial \hat{z}}{\partial z} \quad \text { in } \quad \Omega_{t},
$$

where $\frac{d}{d t}$ denotes the total derivative in the real domain. We will see that it is more convenient to use:

$$
\hat{J}_{t} w^{*}=\hat{w}-\left.\frac{\partial z}{\partial t}\right|_{\hat{\boldsymbol{x}}}-\left.\hat{u} \frac{\partial z}{\partial x}\right|_{\hat{\boldsymbol{x}}}-\left.\hat{v} \frac{\partial z}{\partial y}\right|_{\hat{\boldsymbol{x}}}=\hat{w}-\hat{c}-\left.\hat{u} \frac{\partial z}{\partial x}\right|_{\hat{\boldsymbol{x}}}-\left.\hat{v} \frac{\partial z}{\partial y}\right|_{\hat{\boldsymbol{x}}} .
$$


Remark 3.1 Note that this definition of $w^{*}$, which is standard in the framework of the sigma transformation, can be misleading in the ALE formulation. In particular, the velocity of the domain is contained in this "pseudo vertical velocity". This may explain some confusions found in the literature, and in particular the false idea that models using the sigma transformation do not take into account the movement of the domain.

The expression of the continuity equation (24) in the reference configuration is:

$$
\left.\frac{\partial\left(\hat{J}_{t}\right)}{\partial t}\right|_{\hat{\boldsymbol{x}}}+\left.\frac{\partial\left(\hat{J}_{t} u\right)}{\partial x}\right|_{\hat{\boldsymbol{x}}}+\left.\frac{\partial\left(\hat{J}_{t} v\right)}{\partial y}\right|_{\hat{\boldsymbol{x}}}+\frac{\partial\left(\hat{J}_{t} w^{*}\right)}{\partial \hat{z}}=0 \quad \text { in } \quad \hat{\Omega} .
$$

To obtain this expression, one has to start from equation (24), multiply with the Jacobian determinant $\hat{J}_{t}$, and then use relations (17)-(21).

The momentum equations (22)-(23) can be written in the following non-conservative form:

$$
\left\{\begin{aligned}
&\left.\frac{\partial \hat{\boldsymbol{u}}}{\partial t}\right|_{\hat{\boldsymbol{x}}}+\left.\hat{u} \frac{\partial \hat{\boldsymbol{u}}}{\partial x}\right|_{\hat{\boldsymbol{x}}}+\left.\hat{v} \frac{\partial \hat{\boldsymbol{u}}}{\partial y}\right|_{\hat{\boldsymbol{x}}}+\hat{w}^{*} \frac{\partial \hat{\boldsymbol{u}}}{\partial \hat{z}} \\
&-(\operatorname{div}(\boldsymbol{\nu} \nabla \boldsymbol{u})) \circ \hat{\mathcal{A}}_{t}+\left(\frac{1}{\rho} \boldsymbol{\nabla}_{2} p\right) \circ \hat{\mathcal{A}}_{t}=\hat{f} \quad \text { in } \hat{\Omega} \\
&\left.\frac{\partial \hat{w}}{\partial t}\right|_{\hat{\boldsymbol{x}}}+\left.\hat{u} \frac{\partial \hat{w}}{\partial x}\right|_{\hat{\boldsymbol{x}}}+\left.\hat{v} \frac{\partial \hat{w}}{\partial y}\right|_{\hat{\boldsymbol{x}}}+\hat{w}^{*} \frac{\partial \hat{w}}{\partial \hat{z}} \\
&-(\operatorname{div}(\boldsymbol{\nu} \nabla w)) \circ \hat{\mathcal{A}}_{t}+\hat{J}_{t}^{-1} \frac{1}{\rho} \frac{\partial p}{\partial \hat{z}}=-g \quad \text { in } \hat{\Omega} .
\end{aligned}\right.
$$

But using formulation (32) of the continuity equation, we deduce a conservative form of the momentum equations:

$$
\left\{\begin{aligned}
\left.\frac{\partial\left(\hat{J}_{t} \hat{\boldsymbol{u}}\right)}{\partial t}\right|_{\hat{\boldsymbol{x}}} & +\left.\frac{\partial\left(\hat{J}_{t} \hat{u} \hat{\boldsymbol{u}}\right)}{\partial x}\right|_{\hat{\boldsymbol{x}}}+\left.\frac{\partial\left(\hat{J}_{t} \hat{v} \hat{\boldsymbol{u}}\right)}{\partial y}\right|_{\hat{\boldsymbol{x}}}+\frac{\partial\left(\hat{J}_{t} w^{*} \hat{\boldsymbol{u}}\right)}{\partial \hat{z}} \\
& -\hat{J}_{t}(\operatorname{div}(\boldsymbol{\nu} \boldsymbol{\nabla} \boldsymbol{u})) \circ \hat{\mathcal{A}}_{t}+\hat{J}_{t}\left(\frac{1}{\rho} \boldsymbol{\nabla}_{2} p\right) \circ \hat{\mathcal{A}}_{t}=\hat{f} \text { in } \hat{\Omega}, \\
\left.\frac{\partial\left(\hat{J}_{t} \hat{w}\right)}{\partial t}\right|_{\hat{\boldsymbol{x}}}+\left.\frac{\partial\left(\hat{J}_{t} \hat{u} \hat{w}\right)}{\partial x}\right|_{\hat{\boldsymbol{x}}}+\left.\frac{\partial\left(\hat{J}_{t} \hat{v} \hat{w}\right)}{\partial y}\right|_{\hat{\boldsymbol{x}}}+\frac{\partial\left(\hat{J}_{t} w^{*} \hat{w}\right)}{\partial \hat{z}} & -\hat{J}_{t}(\operatorname{div}(\boldsymbol{\nu} \boldsymbol{\nabla} w)) \circ \hat{\mathcal{A}}_{t}+\frac{1}{\rho} \frac{\partial p}{\partial \hat{z}}=-g \text { in } \hat{\Omega} .
\end{aligned}\right.
$$

Remark 3.2 Note that when the hydrostatic assumption is applied, the system simplifies considerably. Indeed, the accelerations of the vertical velocity $w$ are then neglected and $w$ disappears completely from the transformed equations. In that case, only the newly-defined transformed vertical velocity $w^{*}$ has to be evaluated. The physical vertical velocity can then be recalculated using relation (31). 
As we can see, the expression of the advection terms in a $\sigma$-coordinate system is quite simple using the Jacobian determinant of the ALE mapping. Moreover, it perfectly suits to the formulation of the continuity equation in this system. On the other hand, the kinematic boundary conditions at the top and bottom surfaces of the domain - (25) and (26) - can be expressed in a simple form in the reference configuration; that is

$$
w^{*}=0 \quad \text { at } \hat{z}=0 \text { and } \hat{z}=1 .
$$

Now that we have introduced the ALES approach, we aim to show how it can be helpful to improve the classical variant of the sigma-transformation. In fact, a way to overcome its drawbacks is to design new $\sigma$-coordinate systems, allowing for more adaptability of the vertical discretization. We propose a very general sigma-coordinate system in the ALES approach, which allows for an arbitrary distribution of the layers along the vertical direction at each time step.

\section{GENERALIZATION OF THE SIGMA-TRANSFORMATION IN THE ALES APPROACH}

The idea of developing new sigma-coordinate systems has been considered by meteorologists and oceanographers since the disadvantages of the classical sigma-transformation were detected. In [29], Song and Haidvogel developed the so-called general s-coordinate in order to allow uniformly high resolution near the surface, while maintaining the topography-following properties of the classical sigma-transform. It has proved to be more accurate and stable in the case of a strong stratification, but the Jacobian determinant of this transformation is quite complex, making it difficult to implement. Other authors have focused on systems that would be less sensitive to the pressure gradient error. In [7], Deleersnijder and Beckers proposed to define a fixed horizontal level at a constant depth, splitting the domain into an upper and a lower region, and apply the classical sigma-transformation to both sub-domains. In the case of a slight surface slope, this system leads to almost horizontal levels in the upper region. This idea has also been used in [18] for the simulation of surface flows over submerged structures laid on a flat bottom. In this case, the lower part of the domain remains constant in time, what simplifies the resolution at the structure's surface. This sigma-coordinate system has been generalized in [19] to fit to floating and immersed structures over non-flat bottom. A more general approach was introduced by Gerdes in [10] and consists in assuming a $z$-level distribution on the whole domain and "correcting" locally the depth of a level whenever its distance from the bottom or the surface is smaller than a minimal width chosen arbitrarily.

However, in real domains, the relative importance of topographical effect, density forcing, inertia and diffusion varies from point to point as well as in time. A model should thus allow to use different types of grids for different regions. Therefore, a generic discrete vertical transformation function $Z_{h}$ should be implemented, allowing for arbitrary level distribution at each time step. This idea was first introduced by Deleersnijder and Ruddick in [8], where they applied it to a marine model. Several systems have adopted such a general coordinate system, providing the user with more flexibility in the choice of the optimal model grid for a particular application. Indeed, the recently developed oceanographic ROMS model [27] follows this approach, and a general coordinate system has been introduced for the POM model [25]. They propose a wide variety of particular vertical coordinate systems, including the $z$-level, the 
classical sigma transformation and Gerdes' s-coordinate systems.

Taking advantage of the interpretation of the sigma transformation in the ALES approach, we have developed a very general sigma-coordinate system, allowing for an arbitrary distribution of the layers along the vertical direction, at each time step. Especially, it allows to impose fixed levels in particular zones of the domain, for instance to capture stratifications. The Jacobian determinant of the mapping plays a central role in this system. Indeed, it characterizes the particular transformation defined in each grid element and at each time step, and appears in the expression of the equations driving the flow written in the reference configuration. The system has been implemented in the finite-element-based, three-dimensional free surface flow solver Telemac-3d [12]. We will next describe this general coordinate system, and we will then give some numerical illustration of its advantages.

\subsection{Principle}

Let us consider again the general form (13) of an ALES mapping between the reference configuration $\hat{\Omega}$ and the real domain $\Omega_{t}$ : it is characterized by a continuous and monotonic vertical transformation function $Z: \hat{\Omega} \times I \longrightarrow \Omega_{t}$ satisfying

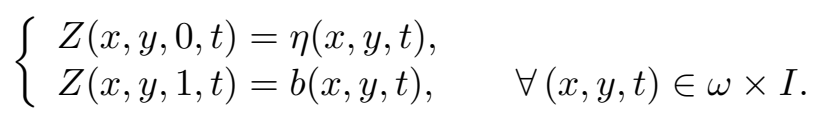

The reference configuration $\hat{\Omega}$ is first discretized by $N_{l}$ horizontal levels, distributed along the vertical following an arbitrary partition of the interval $[0,1]$ :

$$
0=\hat{z}_{1}<\ldots<\hat{z}_{k}<\ldots<\hat{z}_{N_{l}}=1 .
$$

The height of the grid nodes located on the $k$-th level of $\hat{\mathcal{T}}_{h}$ is therefore $\hat{z}_{k}$, which is constant in space and time. We will denote by $l_{k+1 / 2}$ the layer delimited by levels $k$ and $k+1$, for $k=1, \ldots,\left(N_{l}-1\right)$, and by

$$
\Delta \hat{z}_{k+1 / 2}=\hat{z}_{k+1}-\hat{z}_{k}
$$

its width. The transformation function $Z(x, y, \hat{z}, t)$ is then discretized by means of piecewise linear functions in the vertical direction. Inside each layer, this transformation can be expressed as a classical sigma transformation, characterized by the heights - in the real domain - of the levels delimiting the layer. Indeed, the restriction of the discrete transformation on each layer $l_{k+1 / 2}$ can be defined as follows on $\omega \times\left[\hat{z}_{k}, \hat{z}_{k+1}\right] \times I$ :

$$
Z_{k+1 / 2}(x, y, \hat{z}, t)=\frac{\Delta z_{k+1 / 2}(x, y, t)}{\Delta \hat{z}_{k+1 / 2}}\left(\hat{z}-\hat{z}_{k}\right)+z_{k}(x, y, t),
$$

where $z_{k}(x, y, t)$ is an arbitrary discrete function describing the current height of the grid level $k$ at each two-dimensional node and at each time step. Then $\Delta z_{k+1 / 2}(x, y, t)=z_{k+1}(x, y, t)-$ $z_{k}(x, y, t)$ denotes the width of the layer $l_{k+1 / 2}$.

Obviously, it is the choice of the set of discrete functions

$$
\left\{z_{k}(x, y, t) / k=1, \ldots, N_{l}\right\}
$$

which determines the discrete mapping to use for a particular simulation. We point out that they are defined by their value at each node on the two-dimensional mesh and at each time 
step. Moreover, they must satisfy the following conditions for all $(x, y, t) \in \omega \times I$ :

$$
\left\{\begin{array}{rl}
z_{1}(x, y, t) & =b_{h}(x, y, t) \\
z_{N_{l}}(x, y, t) & =h_{h}(x, y, t), \\
z_{k+1}(x, y, t)-z_{k}(x, y, t) & \geq h_{\min }>0
\end{array} \quad \forall k \in\left[1, N_{l}-1\right],\right.
$$

where $h_{\min }$ is a constant minimal width chosen arbitrarily.

Denoting the discrete domain at each time $t$ by $\Omega_{h, t}$, and its triangulation by $\mathcal{T}_{h, t}$, the discrete ALE mapping is defined as follows:

$$
\begin{gathered}
\hat{\mathcal{A}}_{h, t}: \hat{\Omega} \longrightarrow \Omega_{h, t}, \quad \boldsymbol{x}(\hat{\boldsymbol{x}}, t)=\hat{\mathcal{A}}_{h, t}(\hat{\boldsymbol{x}}) \\
\text { with } \quad x=\hat{x}, \quad y=\hat{y} \quad \text { and } \quad z=Z_{h}(x, y, \hat{z}, t),
\end{gathered}
$$

where $Z_{h}(x, y, \hat{z}, t)$ is a continuous function, linear on each layer $l_{k+1 / 2}$ and defined by (37).

This expression of the discrete mapping is very general and allows a wide choice of layer distributions. Indeed, by imposing for $k=1, \ldots,\left(N_{l}-1\right)$

$$
z_{k}(x, y, t)=h_{h}(x, y, t) \hat{z}_{k}+b_{h}(x, y, t)
$$

the classical sigma transformation is recovered. One can also impose some levels to be horizontal and fixed in time at some constant arbitrary height and define classical sigma transformations in between each one of these levels. This can be very useful to catch horizontal stratifications. Such a grid is illustrated in Figure 14. It counts 7 levels - of which 2 are horizontal and fixed in time - and it results from a discrete transformation function $Z_{h}(x, y, t)$ defined from the following set of real level-height functions:

$$
\left\{\begin{array}{rlr}
z_{k}(x, y, t) & =\left(\frac{z_{4}-b_{h}(x, y, t)}{\hat{z}_{4}}\right) \hat{z}_{k}+b_{h}(x, y, t) & \\
z_{4}(x, y, t) & =c_{4} \\
z_{5}(x, y, t) & =c_{5} \\
z_{k}(x, y, t) & =\left(\frac{\eta_{h}(x, y, t)-z_{5}}{1-\hat{z}_{5}}\right)\left(\hat{z}_{k}-\hat{z}_{5}\right)+z_{5} &
\end{array}\right.
$$

where $c_{4}$ and $c_{5}$ are two constant values. But one could as well imagine to impose fixed but non-horizontal levels for the case of steep stratifications.

More generally, the real height of each grid node can be imposed at each time step, defining the discrete function $z_{k}(x, y, t)$ for $k=1, \ldots,\left(N_{l}-1\right)$. The evolution of the grid is then controlled during the whole simulation.

Remark 4.1 We indicate that a test should be made at each time step to make sure that condition (38) is satisfied. In the case the height of a level violates this condition at some node, its value can be corrected using a Min-Mod formula. 


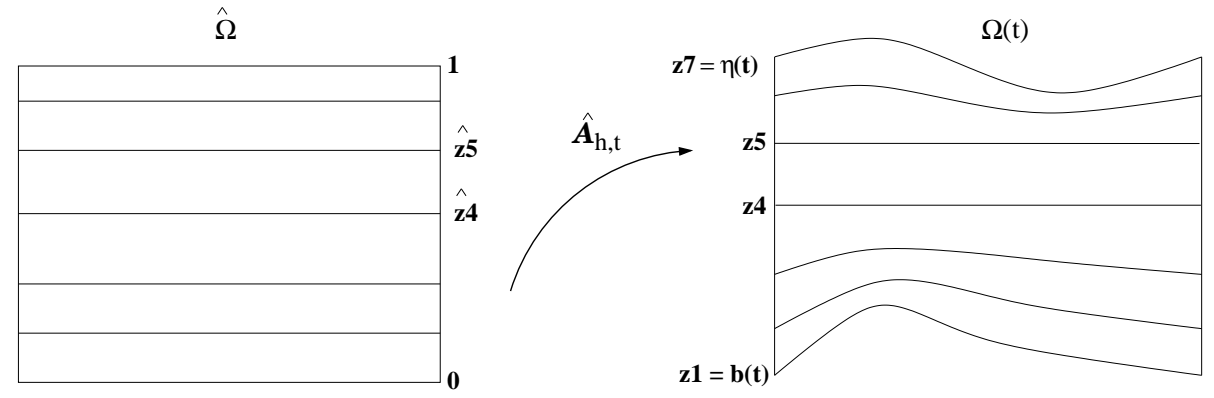

Figure 14: Generalized sigma grid

\subsection{Jacobian determinant of the discrete mapping}

The model must be able to solve the equations for any particular mapping $\hat{\mathcal{A}}_{h, t}$ of the form described previously. Therefore, the Jacobian determinant $\hat{J}_{h, t}$ of the discrete mapping must be expressed in a generic form.

We recall that the Jacobian determinant of the classical sigma transformation is constant along the vertical and equal to the water depth. This is no longer true when the transformation is generalized as it is here. Indeed, in this case, $\hat{J}_{h, t}$ varies along the vertical direction but it remains constant on each grid layer in the vertical direction. Its expression on each layer $l_{k+1 / 2}$, for $k=1, \ldots,\left(N_{l}-1\right)$ is

$$
\begin{aligned}
\hat{J}_{h}(x, y, \hat{z}, t) & =\frac{\partial Z_{k+1 / 2}}{\partial \hat{z}}(x, y, \hat{z}, t)=\frac{\Delta z_{k+1 / 2}(x, y, t)}{\Delta \hat{z}_{k+1 / 2}} \\
& \text { in } \omega \times\left[\hat{z}_{k}, \hat{z}_{k+1}\right], \quad \forall t \in I .
\end{aligned}
$$

In the following, we will denote by $\hat{J}_{k+1 / 2}$ the restriction of $\hat{J}_{h}$ to layer $l_{k+1 / 2}-$ in which the jacobian determinant is independent of the $\hat{z}$-coordinate:

$$
\hat{J}_{k+1 / 2}(x, y, t)=\frac{\Delta z_{k+1 / 2}(x, y, t)}{\Delta \hat{z}_{k+1 / 2}} \quad \text { in } \quad \omega, \quad \forall t \in I .
$$

The integral of any discrete function $\psi_{h}: \Omega_{h, t} \longrightarrow \mathbb{R}$ on the discrete domain $\Omega_{h, t}$ can thus be transformed and decomposed as follows:

$$
\begin{aligned}
\int_{\Omega_{h, t}} \psi_{h} d \boldsymbol{x} & =\int_{\hat{\Omega}} \hat{J}_{h, t} \hat{\psi}_{h} d \hat{\boldsymbol{x}} \\
& =\sum_{k=1, \ldots, N_{l}-1} \int_{\hat{l}_{k+1 / 2}} \hat{J}_{k+1 / 2}(x, y, t) \hat{\psi}_{h}(x, y, \hat{z}) d \hat{\boldsymbol{x}} \\
& =\sum_{k=1, \ldots, N_{l}-1} \int_{\omega} \hat{J}_{k+1 / 2}(x, y, t)\left(\int_{\hat{z}_{k}}^{\hat{z}_{k+1}} \hat{\psi}_{h}(x, y, \hat{z}, t) d \hat{z}\right) d \omega .
\end{aligned}
$$

The value of this discrete Jacobian determinant characterizes the particular transformation defined in each grid element and at each time step. It appears in the discrete counterpart of the equations driving the flow when written in the reference configuration. 


\section{NUMERICAL ILLUSTRATION}

The general sigma-coordinate system introduced previously has been implemented in the finiteelement-based, three-dimensional free surface flow solver Telemac-3d [12], in order to allow more flexibility in the choice of the optimal vertical domain discretization. In this last part of the paper we aim to show numerically some advantages of this new coordinate system. For this purpose we present three test cases, for which we compare the results obtained with the Telemac-3d system using different grids. Some of those grids can be defined using the classical sigma transformation, others must be defined using the generalized version.

\subsection{Test case 1: density stratification in a standing basin}

We consider again the test of the standing basin with a density stratification presented in section 2.3. We now perform the same simulation on a grid obtained using the generalized sigma transformation. It still counts the same number of levels, 11, but they are distributed differently: a fixed plane is imposed exactly at the height of the stratification, and a homogeneous classical sigma transformation is operated in both the lower and the upper parts of the basin. Figure 15 shows left the resulting mesh and right the density distribution obtained after 100 seconds with this mesh.
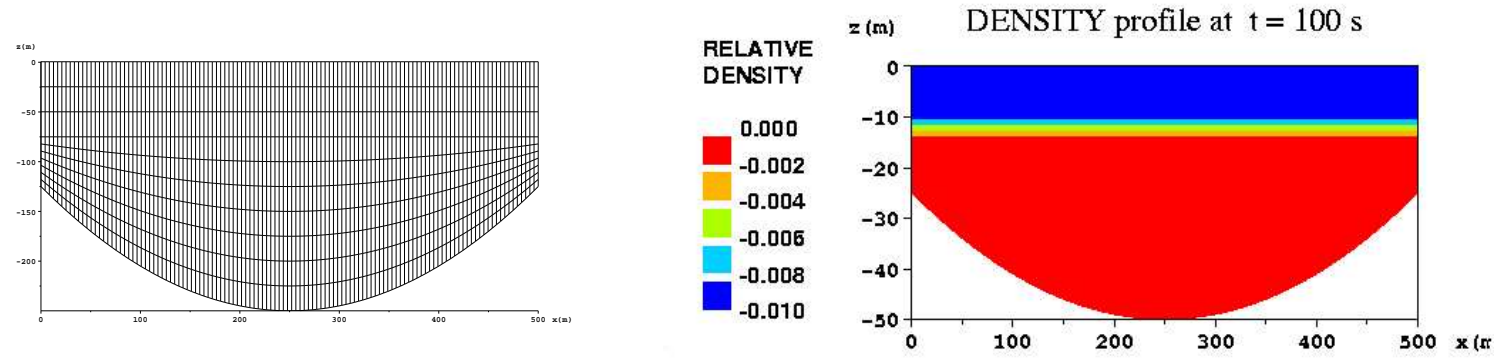

Figure 15: Generalized-sigma transformation mesh (left) and density distribution (right) obtained at $t=100 \mathrm{~s}$. Vertical cross-sections at $y=50 \mathrm{~m}$.

We observe that the stratification is unperturbed. In addition, figure 16 shows that no spurious velocities have appeared, even after 1000 seconds. Thus, the equilibrium has been completely preserved. This shows that fixed planes can help to reproduce accurately density stratifications.

Of course this solution is ad hoc since the mesh fits exactly to the shape of the stratification. Nevertheless, in some practical applications, this shape and its time evolution may be known, at least approximately. In such cases, the ALES formulation allows to adapt the mesh in order to reproduce accurately the stratification, getting rid of strong spurious diffusion obtained with the classical sigma transformation technique.

\subsection{Test case 2: advection of a passive tracer in a $3 \mathrm{D}$ closed basin with a standing wave.}

In this test case we aim to reveal the amplification of the numerical diffusion due to the movement of the mesh, and show that it is possible to minimize this phenomenon by fixing some 


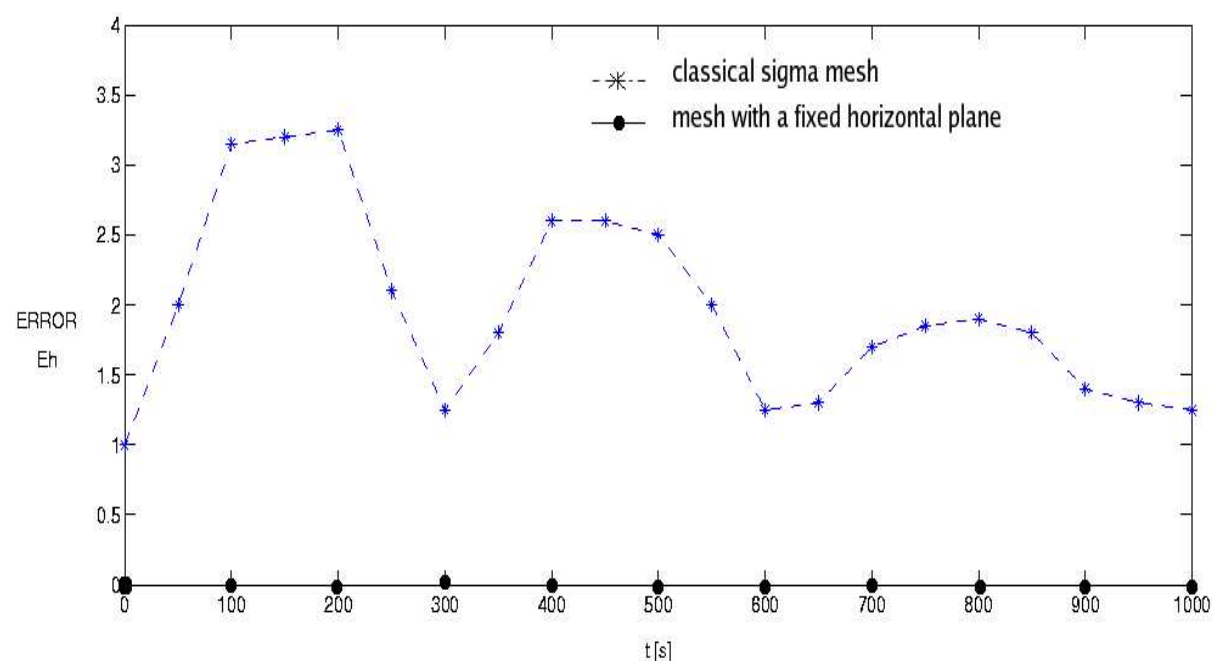

Figure 16: Time evolution of the error $E_{h}$ in the case of an initial horizontal density stratification.

particular zones of the mesh.

For this purpose we consider the case of the standing wave in a closed basin. The fluid is inviscid, and a passive tracer - which has no influence on the hydrodynamics of the fluid - is advected by the flow. A closed square basin with side $L$ and with a constant bottom at $z=-H$ is considered, where $H$ is the equilibrium water depth. In order to concentrate on the advection of the tracer, we impose the analytical solution of the flow, provided by the small amplitude wave theory for the linear equations - see for instance [16]. The analytic free-surface profile and fluid velocities are defined as follows:

$$
\left\{\begin{array}{l}
\eta=\eta_{0} \cos (k \pi x) \cos (\omega t), \\
u=\omega \eta_{0} \frac{\cosh (k \pi(z+H))}{\sinh (k \pi H)} \sin (k \pi x) \sin (\omega t), \\
v=0, \\
w=-\omega \eta_{0} \frac{\sinh (k \pi(z+H))}{\sinh (k \pi H)} \cos (k \pi x) \sin (\omega t),
\end{array}\right.
$$

with $\omega^{2}=g k \tanh (k H)$, where $\eta_{0}=0.2$ is the amplitude of the wave and $k=1 / L$ the frequency. The analytical water depth profile at $x=0 \mathrm{~m}$ is represented in Figure 17.

At the initial time, the tracer $T$ is stratified along the vertical: its concentration $c$ is equal to 4 if $z \geq-4.5 \mathrm{~m}$, and it is equal to 2 if $z<-4.5 \mathrm{~m}$. We emphasize the fact that there is no diffusion on the tracer, only advection. The perturbation of the stratification should therefore not be larger than the wave amplitude of the flow. A first simulation is performed on the threedimensional grid represented left in Figure 18, obtained using the classical sigma transformation with 11 homogeneously distributed levels on the vertical. Figure 18 presents right the vertical 


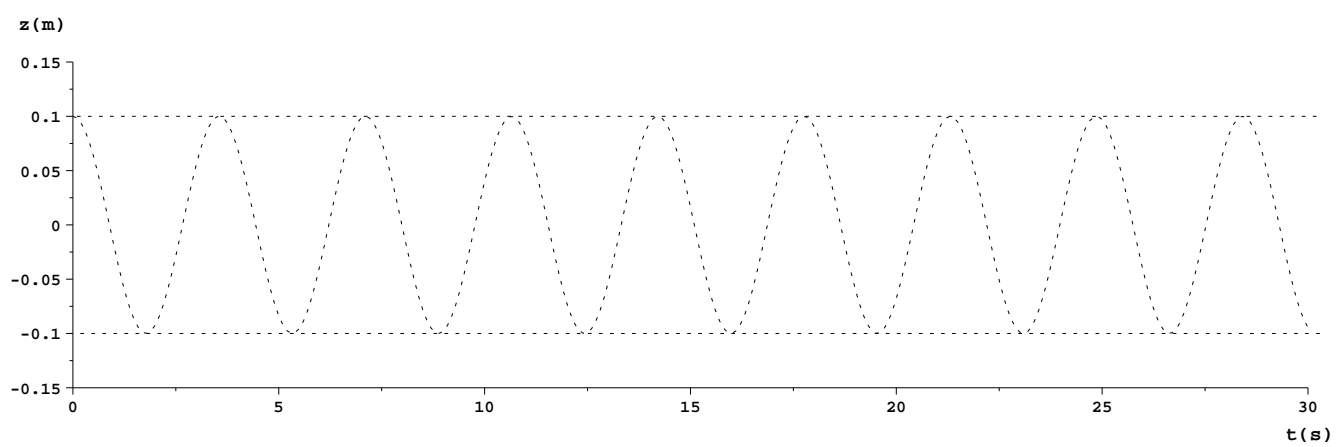

Figure 17: Temporal profile of the exact water depth at $x=0 \mathrm{~m}, y=5 \mathrm{~m}$.

tracer distribution obtained after 30 seconds of simulation : we observe an important vertical diffusion of the tracer distribution. The precision of the simulation can be improved by refining
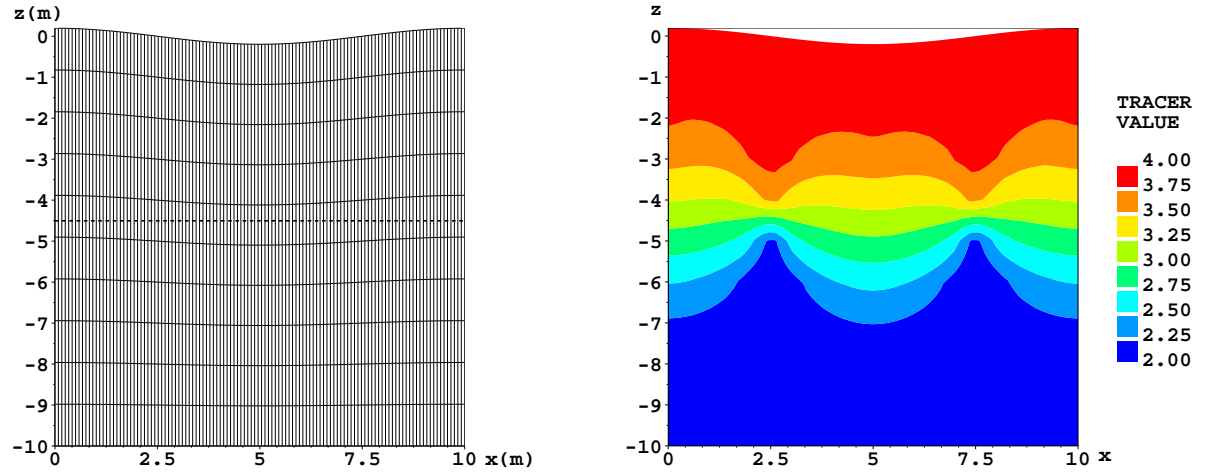

Figure 18: Classical sigma mesh (left) and tracer profile obtained at $t=30$ s (right) with this mesh.

the mesh in the stratified zone. This mesh, represented left in Figure 19, is obtained using the classical sigma transformation with the same number of levels, but using a non-homogeneous distribution of these levels along the vertical. The tracer distribution obtained after 30 seconds is shown right in Figure 19: it is slightly less diffused.

Now, in order to evaluate the precision of these results, we have performed the same simulation refining significantly the mesh along the vertical: we have used 55, 105 and finally 205 levels on the vertical. Figure 20 presents the results of these simulations after 30 seconds. They show that the discrete solution converges to a solution in which the perturbation of the stratification is of the same order as the wave amplitude.

Let us now evaluate the effect of the mesh movement on the result of this simulation. We use a new grid, shown left in Figure 21 and obtained using the generalized sigma transformation described in this paper. It counts 11 levels and the vertical resolution in the stratified zone is the same as in the second classical-sigma mesh (represented left in Figure 19), but a horizontal plane is fixed at height $z=-3.5 \mathrm{~m}$, so that the inferior part of the mesh does not move. The results obtained on this grid are presented right in figure 21 .

The classical sigma mesh shown in Figure 19 and the generalized sigma mesh used now have the 

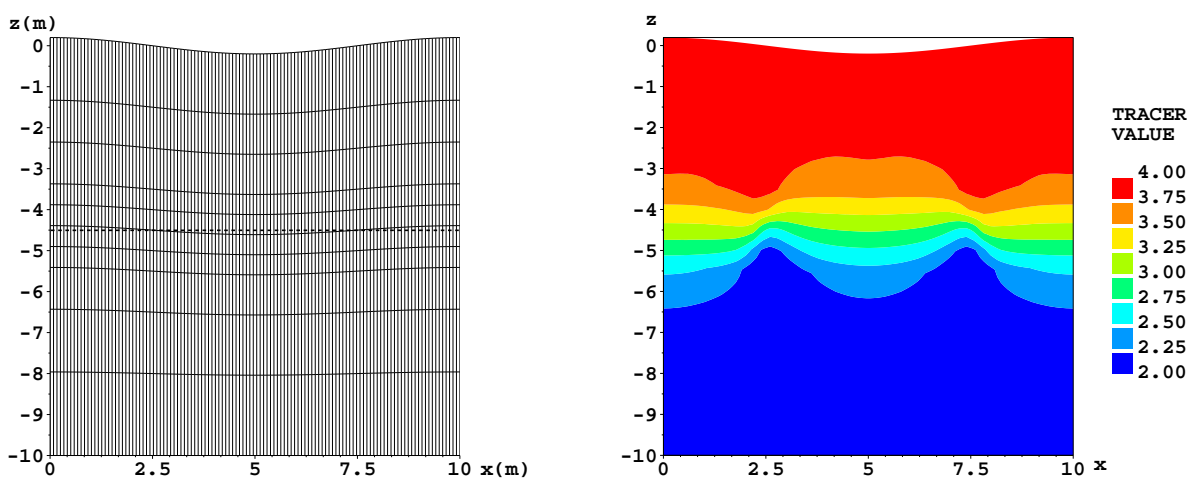

Figure 19: non-homogeneous classical sigma mesh (left) and tracer profile obtained at $t=30$ s (right) with this mesh.

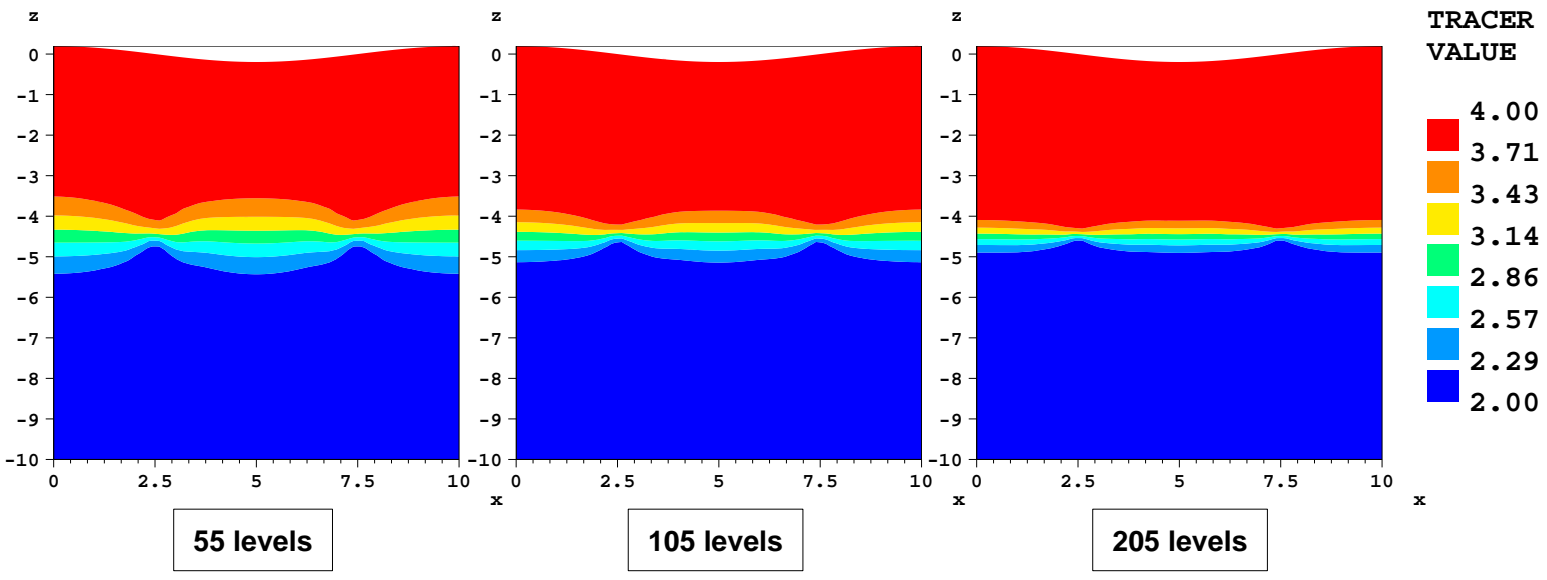

Figure 20: Tracer profile obtained at $t=30 \mathrm{~s}$ increasing the number of levels.
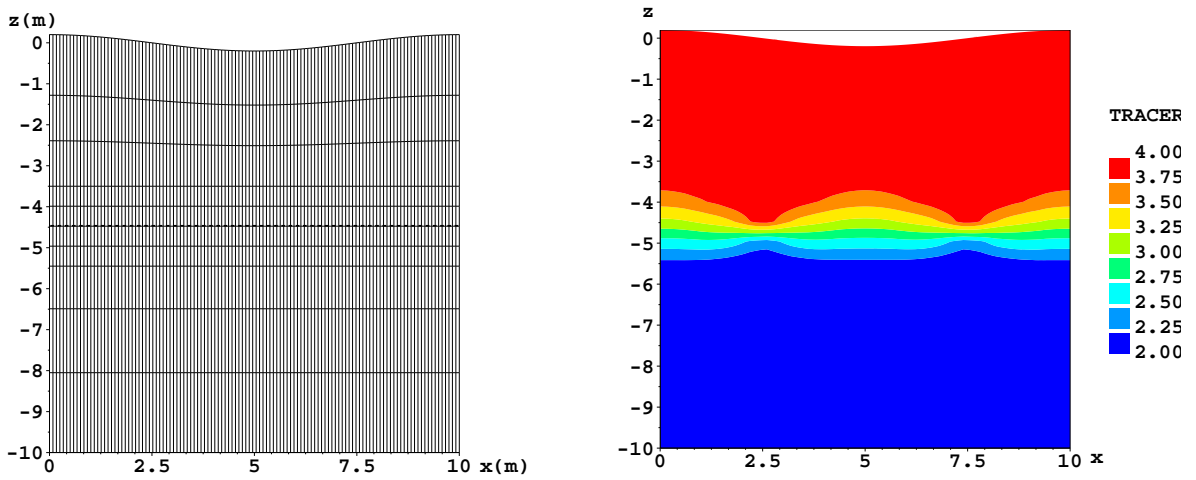

Figure 21: Generalized sigma-transformation mesh (left) and tracer profile (right) obtained at $t=30$ s with this mesh. 
same vertical resolution, but in the first case all the layers move with the free surface, whereas in the second case only the upper layers move. Comparing the results obtained on both meshes we observe that the vertical diffusion of the tracer distribution is significantly smaller when using the second mesh : the movement of the mesh in the vertical direction amplifies the vertical numerical diffusion. In fact, the result obtained with a mesh counting 11 layers, from which 8 are fixed, is as accurate as the result obtained with a mesh counting 55 moving layers (see Figure 20).

This test case shows that the movement of the mesh can sometimes provoke additional numerical diffusion. This can be limited using the ALES transformation, by imposing fixed layers and therefore cancelling the movement of the mesh in particular zones that should be solved more accurately.

\subsection{Test case 3: Effect of a heat source in a cone-shaped basin.}

We now show some results on a realistic test case, which simulates the effect of a heat source in the middle of a closed basin with steep bathymetry. This test case does not have an analytical solution, and we do only present qualitative results. However, in this case the bottom gradient has no influence on the heat spreading, and we should therefore obtain the same temperature distribution as in the case where the bottom is flat. We therefore begin with a simulation of the same test case with a flat bottom, and we then compare it to the results obtained in the case of steep bathymetry, using two different vertical discretizations.

We first consider a square closed basin of side $L=4000 \mathrm{~m}$, with a constant bottom at $z=-10 \mathrm{~m}$. At the initial time the water surface is flat $(h=10 \mathrm{~m})$ and no motion is taken. Figure 22 shows the vertical cross-section of the initial three-dimensional mesh at $y=2000 \mathrm{~m}$. After one time step, water is discharged with an excess temperature of $T=333^{\circ} \mathrm{C}$ from a point

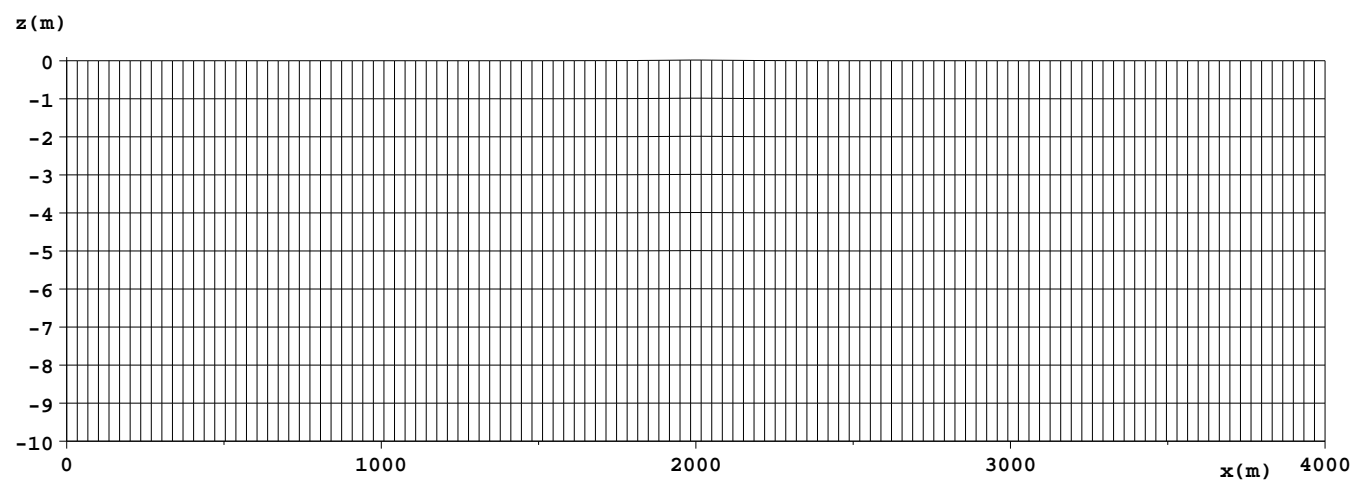

Figure 22: Vertical cross-section at $y=2000 \mathrm{~m}$ of the 3D mesh.

source located in the middle of the water column at the centre of the basin. The coordinates of this source point are $x=2000 \mathrm{~m}, y=2000 \mathrm{~m}$ and $z=-5 \mathrm{~m}$. The water discharge of the source is $Q=20 \mathrm{~m}^{3} / \mathrm{s}$. A constant horizontal viscosity coefficient of $\nu_{h}=10^{-4}$ and no vertical diffusion are chosen for the fluid velocities. No diffusion at all is considered on the tracer, so that only the advection is evaluated. We indicate that the temperature is an active tracer: the equation of state for perfect gases relates the fluid density to the temperature. The temperature profile after 30 minutes is shown in Figure 23. We can see that the effect of the heat on the flow has been taken into account : the velocities are mainly vertical and they are oriented in 
the direction of the free surface since the heat goes upwards.

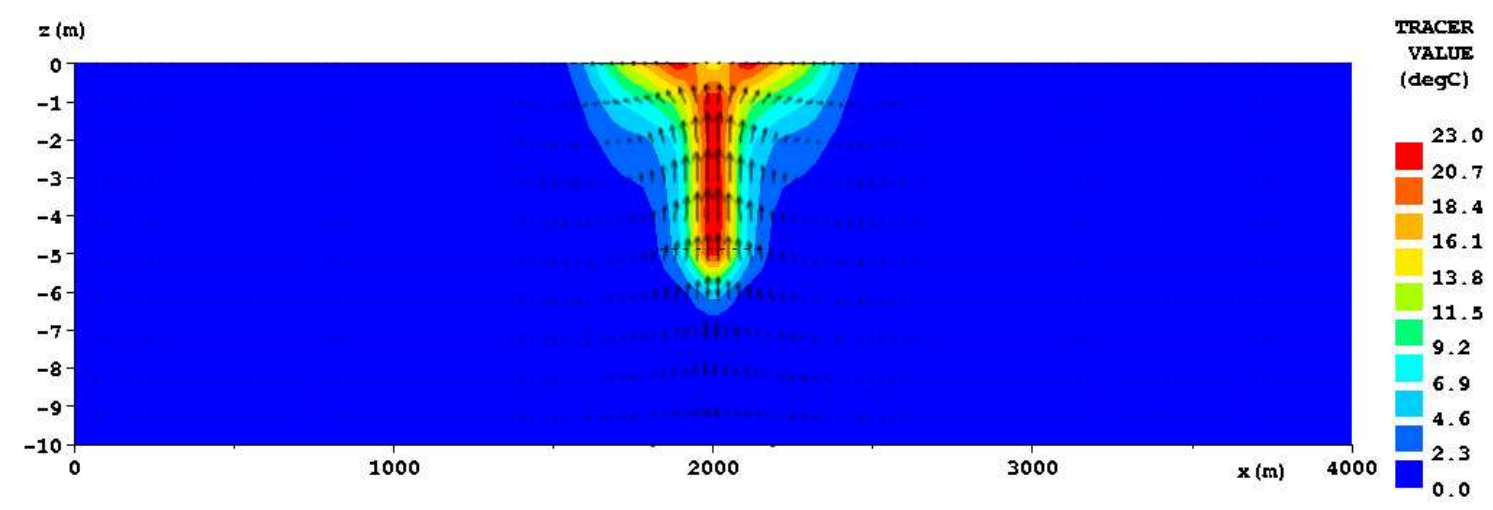

Figure 23: Tracer distribution at $t=1800 \mathrm{~s}$.

We now consider a different configuration. A cone-shaped obstacle is placed on the bottom at the middle of the domain. We want to compare the results obtained on two different meshes, whose vertical cross-sections are shown in Figure 24 : mesh (a) has been obtained using the classical sigma transformation whereas mesh (b) has been obtained using Gerdes transformation [10], which can be defined through the generalized sigma coordinate system introduced in this paper. This transformation allows to have nearly horizontal layers everywhere. In that way, the layers containing the heat source do not move during the entire simulation, and they are almost horizontal. In mesh (a) instead, the layers around the source move with the free surface, and they contain highly-deformed elements, some of which do not fulfil the hydrostatic consistency condition. We have no analytical solution for this test case. However, the obstacle on the bottom should have no consequence on the tracer distribution. Therefore, we expect to obtain the same result as in the previous simulation with flat bottom. Figure 25 shows the temperature profiles obtained after 360 time steps, using both meshes, as vertical-cross sections at $y=2 \mathrm{~m}$. The tracer distribution obtained with the classical sigma mesh (a) is very different from the one obtained on a flat bottom (see figure 23). The temperature has a maximal value of 38 degrees in the middle of the basin, whereas it doesn't exceed 23 degrees in the case of the flat bottom. In addition, a non-physical effect appears around the upper part of the obstacle: the heat spreads out in the bottom direction, whereas it should only go upwards. On the contrary, with mesh (b), the tracer distribution is almost the same as the one obtained with a flat bottom. Figure 26 gives a better comparison of both tracer profiles. We see that the shape and also the values of the tracer distribution are very similar. We can therefore say that the result obtained using the generalized sigma mesh (b) is more accurate.

Imposing nearly horizontal layers in areas with density gradients can improve the accuracy of the simulations. Therefore, it is advantageous to have more flexibility in the definition of the vertical discretization, as it is the case with the generalized sigma coordinate system we have proposed in this paper.

\section{CONCLUSION}

In this paper, the link between the sigma transform approach and the ALE approach has been clearly established. Indeed, we have shown that the sigma transform is equivalent to the ALE approach with a particular type of mappings. We have therefore introduced the ALE-Sigma (ALES) approach, which consists in an ALE interpretation of the sigma transform. Then, 


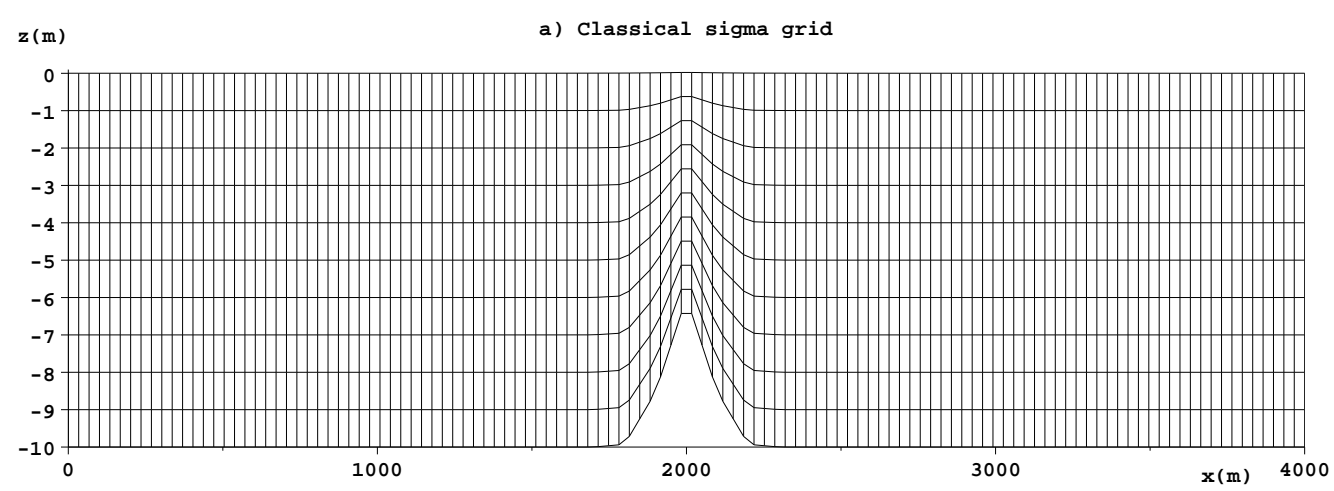

$\mathbf{z}(\mathrm{m})$

b) Generalized sigma grid

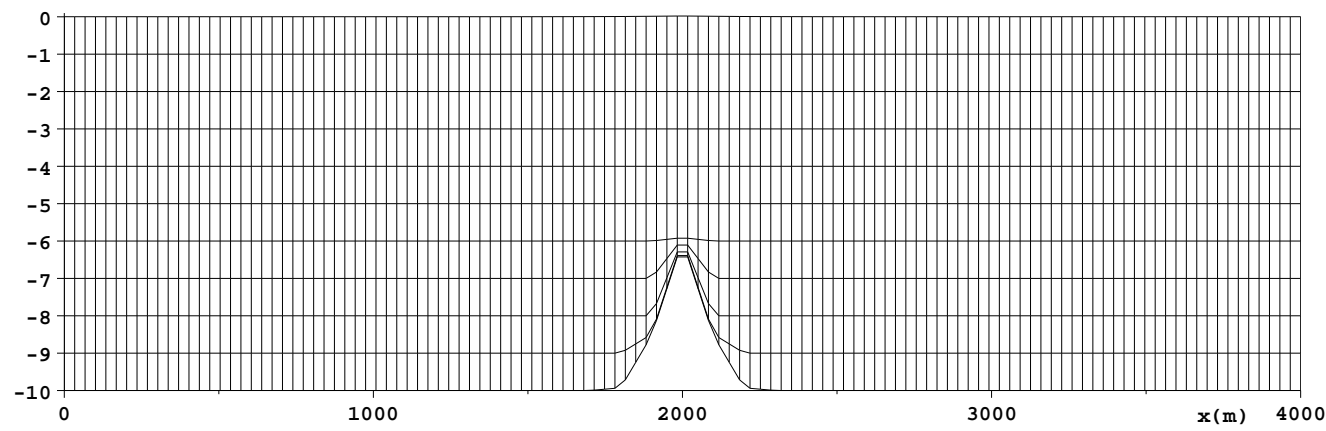

Figure 24: Mesh (a) has been obtained using the classical sigma transformation. Mesh (b) has been obtained using a more general sigma coordinate system.

we have proposed a very general transformation in the ALES approach, allowing for a great adaptability of the vertical discretization and therefore overcoming some drawbacks of the classical sigma transform. This general sigma-coordinate system has been implemented in the three-dimensional free surface flow solver Telemac-3d [12], in order to allow more flexibility in the choice of the optimal vertical domain discretization. Several numerical tests have shown the advantages of this new coordinate system, as for example a better representation of horizontal stratifications.

Acknowledgements: This work was supported by the LNHE laboratory, EDF (electricity of France), in the framework of a PhD Thesis. We would like to thank Jean-Michel Hervouet and Emile Razafindrakoto for their collaboration on this work. The numerical tests were performed with the three-dimensional free surface flow solver Telemac-3d developed at the LNHE, EDF.

\section{References}

[1] Beckman A. and Haidvogel D., Numerical simulation of flow around a tall isolated seamount. J. Phys. Oceanogr. 23: 1736-1753, 1993.

[2] Blumberg A.F. and Mellor G.L., A description of a three-dimensional coastal ocean circulation model. In N. Heaps (ed.), Three-dimensional Coastal Ocean Models, Vol.4, American Geophysical Union, Washington D.C., 1-16, 1987. 


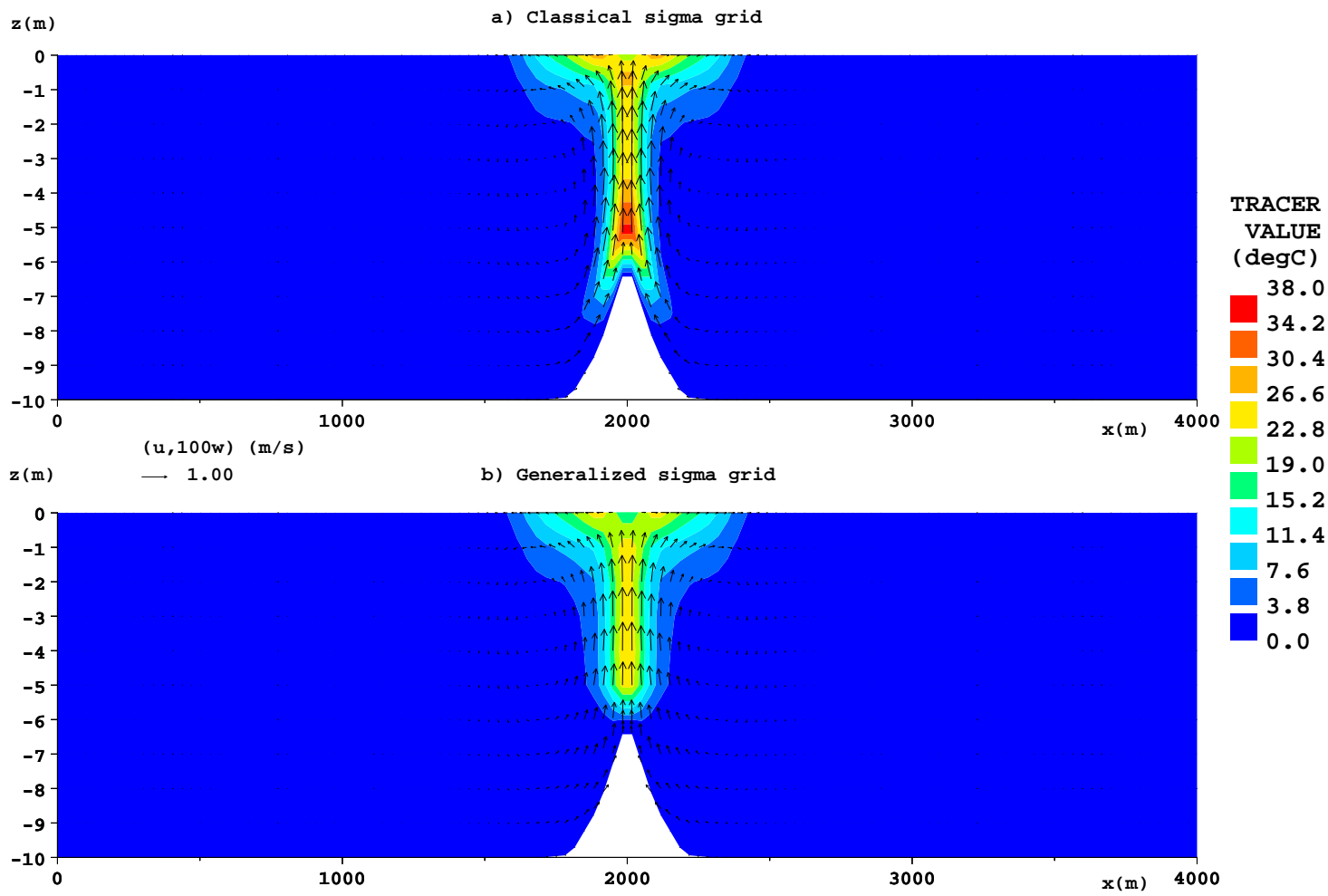

Figure 25: Temperature profiles obtained at $t=1800 \mathrm{~s}$ using (a) the classical sigma mesh and (b) the sigma generalized mesh.

[3] Bryan K., A numerical model for the study of the circulation of the world oceans. $J$. Comput. Phys. 4: 347-376, 1969.

[4] Burchard H. and Petersen O., Hybridization between $\sigma$-and $z$-coordinates for improving the internal pressure gradient calculation in marine models with steep bottom slopes. Int. J. Numer. Methods Fluids 25: 1003-1023, 1997.

[5] Chu P.C., Fan C., Hydrostatic correction for sigma coordinate ocean models J. Geophysical Research 108 (C6), 3206, 2003.

[6] Decoene A., Hydrostatic model for three-dimensional free surface flows and numerical schemes. PhD thesis, Université Pierre et Marie Curie, Paris VI, 2006.

[7] Deleersnijder E. and Beckers J.M., On the use of the $\sigma$ coordinate system in regions of large bathymetric variations. J. Marine Syst. 3: 381-390, 1992.

[8] Deleersnijder E. and Ruddick K.G., A generalized vertical coordinate for 3D marine models. Bull. Soc. R. Sci. Liège 61(6): 498-502, 1992.

[9] Gary J.M., Estimate of truncation error in transformed coordinate primitive equation atmospheric models. J. Atmos. Sci. 30: 223-233, 1973.

[10] Gerdes R., A primitive equation ocean circulation model using a general vertical coordinate transformation. Journal of Geophysical Research 98, 14: 683-701, 1993. 

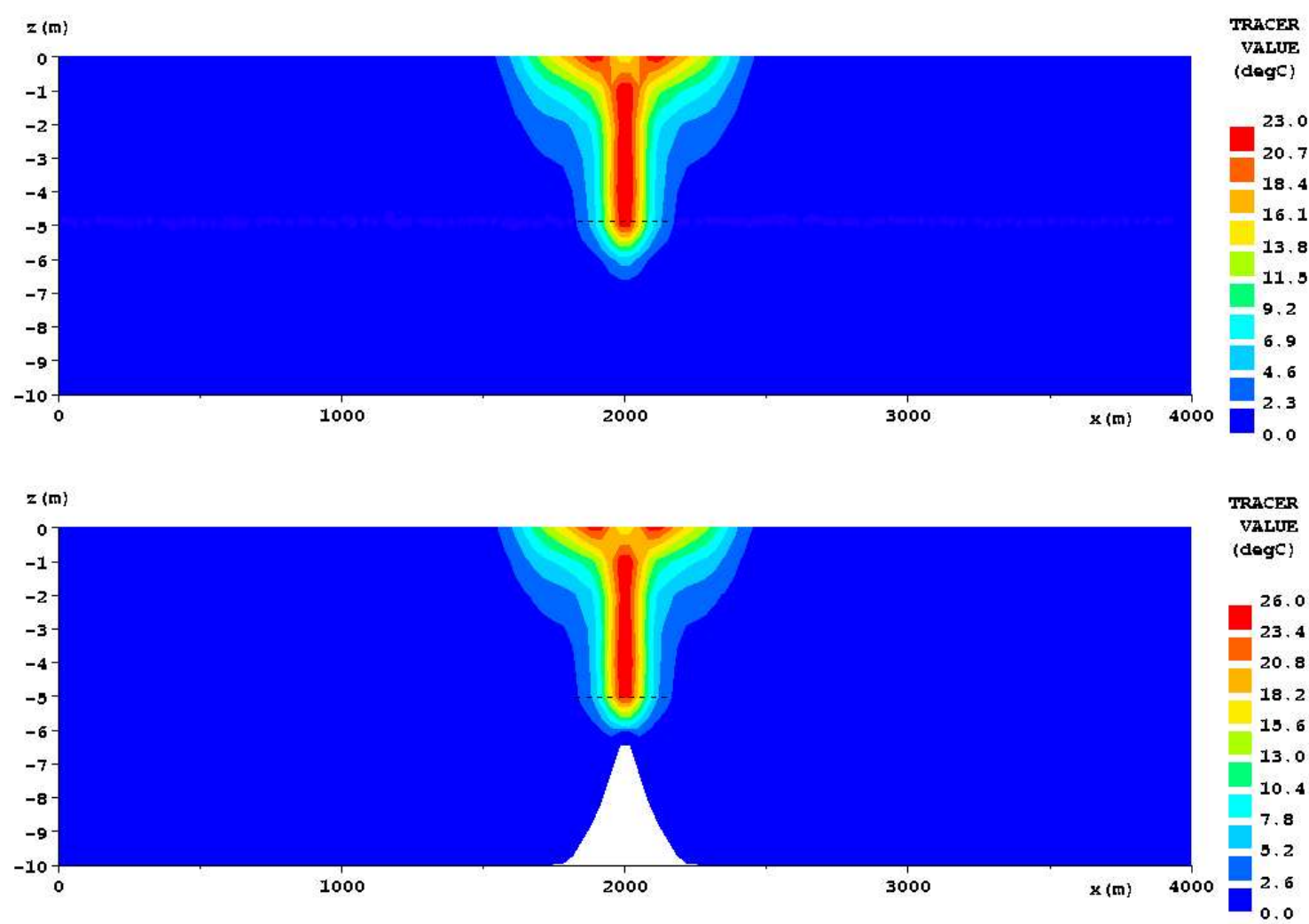

Figure 26: Temperature profiles obtained at $t=1800 \mathrm{~s}$ with flat bottom (upper figure) and with the obstacle on the bottom and using the generalized sigma mesh.

[11] Haney T.L., On the pressure gradient force over steep topography in sigma coordinate ocean models. J. Phys. Oceanogr. 21: 610-619, 1991.

[12] Hervouet J.-M., Hydrodynamique des écoulements à surface libre. Modélisation numérique avec la méthode des éléments finis. Presses de l'école nationale des Ponts et Chaussées, 2003.

[13] Hugues T.J.R., Lagrangian-Eulerian finite element formulation for incompressible viscous flows. Comput. Methods Appl. Mech. Engrg. 29: 329-349, 1981.

[14] Huwald H., Tremblay L.-B., Blatter H., A multi-layer sigma-coordinate thermodynamic snow sea-ice model: Validation against Surface Heat Budget of the Arctic Ocean (SHEBA) / Sea Ice Model Intercomparison Project (SIMIP2) data., J. Geophys. Res., 110(C5), doi: 10.1029/2004JC002328, 2005.

[15] Janjic Z.I., Pressure gradient force and advection scheme used for forecasting with steep and small scale topography. Contrib. Atmos. Phys. 50: 186-199, 1977.

[16] Jankowski J.A., A non-hydrostatic model for free surface flows. PhD thesis, UniversitÂd't Hannover, Institut fãijr StrÃúmungsmechanik und ERiB, 1998.

[17] Kocyigit M., Falconer R., Lin B., Three-dimensional numerical modeling of free surface flows with non-hydrostatic pressure. Int. J. Numer. Methods Fluids 40: 1145-1162, 2002. 
[18] A sigma coordinate 3D kappa-epsilon modelfor trubulent free surface flow over a submerged structure, Applied mathematical modeling 26, 1139-1150, 2002.

[19] Lin P., A multiple-layer sigma-coordinate model for simulation of wave-structure interaction. Computers and Fluids 35: 147-167, 2005.

[20] Malcherek A., Mathematische modellierung von Str $\widetilde{d}$ 'mungen und Stofftransportprozessen in estuaren. PhD thesis, Universit Ãd't Hannover, Institut f̃̃ijr StrÃúmungsmechanik und ERiB, 1995.

[21] Marcos F., Janin J.M., Nouveaux dÃl'veloppements dans l'Ãl'tape de convection-diffusion de Telemac-3D. Rapport EDF R\&D-LNHE, HE-42/94/025/A. 1997.

[22] McCalpin J.D., A comparison $\mathrm{f}$ second-order and fourth-order pressure gradient algorithm in a sigma-co-ordinate model. Int. J. Numer. Methods Fluids 18: 361-383, 1994.

[23] Mellor G.L., Ezer T. and Oey L.-Y., The pressure gradient conundrum of sigma coordinate ocean models. J. Atmosph. Oceanic. Technol. 11: 1126-1134, 1994.

[24] Mellor G.L., Oey L.-Y. and Ezer T., Sigma Coordinate Pressure Gradient and the Seamount Problem. J. Atmosph. Oceanic. Technol. 15: 1122-1131, 1997.

[25] Mellor G., Hakkinen S., Ezer T. and Patchen R. A Generalization of a Sigma Coordinate Ocean Model and an Intercomparison of Model Vertical Grids. Ocean Forecasting: Conceptual Basis ans Applications. N. Pinardi, J. Woods (Eds.), Springer Verlag, Berlin, 55-72, 2002 .

[26] Phillips N.A., A coordinate system having some special advantages for numerical forecasting. J. Meteorol. 14: 184-185, 1957.

[27] Shchepetkin A.F., McWilliams J.C., The Regional Ocean Model System (ROMS) : A split-explicit, free-surface, topography-following coordinate ocean model. Ocean Modelling 9: 347-404, 2005.

[28] Slordal L.H, The pressure gradient force in sigma-coordinate- ocean models. Int. J. Numer. Meth. Fluids 24: 987-1017, 1997.

[29] Song Y. and Haidvogel D., A Semi-implicit Ocean Circulation Model Using a Generalized Topography-Following Coordinate System. J. of Comput. Phys. 115: 228-244, 1994.

[30] Song Y. and Haidvogel D., A general pressure gradient formulation for ocean models. Monthly Weather Review 126: 1749-1762, 1998.

[31] Soulaïmani A., Saad Y., An arbitrary Lagrangian-Eulerian finite element method for solving three-dimensional free surface flows. Comput. Methods Appl. Mech. Engrg. 162: 79-106, 1998.

[32] Zhou J.G., Stansby P.K., An arbitrary Lagrangian-Eulerian Sigma (ALES) model with non-hydrostatic pressure for shallow water. Comput. Methods Appl. Mech. Engrg. 178: 199-214, 1999.

[33] Stelling G.S. and Van Kester J.A.TH.M., On the approximation of horizontal gradients in sigma-coordinates for bathymetry with steep bottom slopes. Int. J. Numer. Methods Fluids 18: 915-935,1994. 
[34] Yuan H., Wu C.H., A two-dimensional vertical non-hydrostatic $\sigma$ model with an implicit method for free-surface flows. Int. J. Numer. Meth. Fluids 44: 811-835, 2004. 